\title{
Postsynaptic Inhibition Mediates High-Frequency Selectivity in the Cricket Teleogryllus oceanicus: Implications for Flight Phonotaxis Behavior
}

\author{
Thomas G. Nolen and Ronald R. Hoy \\ Section of Neurobiology and Behavior, Cornell University, Ithaca, New York 14853
}

The frequency selectivity of the identified auditory interneuron, Int-1, in the cricket Teleogryllus oceanicus was examined using intracellular recording and staining techniques. Previous behavioral assays showed that crickets discriminate the low frequencies of the species calling song (4-5 $\mathrm{kHz}$ ) from the high frequencies contained in the vocalizations of insectivorous bats (Nolen and Hoy, 1986a). Int- 1 was excited by frequencies between 3 and $40 \mathrm{kHz}$, being similar, therefore, to the tympal organ (ear) in its broad range sensitivity; however, it responded differentially to high and low frequencies in terms of (1) the number of action potentials evoked per stimulus tone pulse, (2) the average discharge rate, and (3) the latency of response. It was especially responsive to ultrasound ( $>20 \mathrm{kHz}$ ), discharging at rates up to 400 spikes/sec (average rate), with $10 \mathrm{msec}$ latencies; its response to pulses of the calling song was less than 150 spikes/sec, with $30 \mathrm{msec}$ latencies. Int-1's dynamic range for ultrasound was also quite large, about $50 \mathrm{~dB}$, compared to $\mathbf{2 0} \mathrm{dB}$ for the calling song frequency. In addition, it responded well to trains of short, batlike pulses of ultrasound. These results are consistent with previous behavioral experiments showing that during flight, Int-1 was both necessary and sufficient for the ultrasound avoidance steering behavior (Nolen and Hoy, 1984), as long as it discharged above a rate of 180 spikes/sec. Ultrasound readily produced such high rates, whereas calling song rarely did; ultrasound reliably evoked avoidance steering over a wide dynamic range, while tone pulses of the calling song rarely did so (Nolen and Hoy, 1986a).

A unique source of ipsilaterally mediated inhibition, tuned to the calling song frequency, accounted for (1) the poor response to calling song and hence the neuron's high-frequency selectivity, and (2) the behavioral and physiological effects of 2-tone suppression of high frequencies by the calling song (Nolen and Hoy, 1986b). These results further strengthen Int-1's proposed role as a "bat-detector" during flight and suggest only a limited role in other contexts such as social behavior.

Received July 29, 1985; revised Jan. 2, 1987; accepted Jan. 8, 1987.

We thank J. Hauptman, S. Rollins, D. Yager, L. Nolen, and Al. Cohen for excellent technical assistance, and Drs. R. Harris-Warrick, C. Hopkins, S. Volman, and C. Rankin for helpful comments on the manuscript. We also thank Dr. W. W. Stewart for providing us with a sample of Lucifer yellow. This work was supported by NINCDS research grants and an RCDA to R.R.H. and by an NIGMS pre-doctoral training grant to T.G.N.

Correspondence should be addressed to Thomas G. Nolen, Yale University, Department of Psychology, Box 11A, Yale Station, New Haven, CT 06520.

Copyright $(C) 1987$ Society for Neuroscience 0270-6474/87/072081-16\$02.00/0
Many animals are sensitive to airborne sound in their environment and can locate potential mates or rivals and detect possible predators by acoustic means. In general, auditory systems must discriminate important, biologically relevant signals from irrelevant background noise, determine the location of the stimulus in space, and provide sufficient information to allow the animal to take the appropriate action (Suga, 1973, 1978; Evans, 1975).

The acoustic abilities of insects have long been known (Regen, 1913; Schaller and Timm, 1950; Autrum, 1963; Roeder, 1967), and while their auditory systems are relatively simple, some are capable of analyzing complex sounds (Michelsen, 1971a, b; Miller, 1971; Rheinlaender, 1975; Hill and Boyan, 1977; Boyan, 1980, 1981; Oldfield, 1982; Boyd and Lewis, 1983; Moiseff and Hoy, 1983; Boyd et al., 1984; Nolen and Hoy, 1986b). For example, a variety of biophysical and physiological studics have demonstrated the potential for frequency discrimination by insects (Michelsen, $1971 \mathrm{a}$, b; Rheinlaender, 1975; Boyan, 1981; Oldfield, 1982; Stephen and Bennet-Clark, 1982; Moiseff and Hoy, 1983; Hutchings and Lewis, 1984; Oldfield et al., 1986). Frequency discrimination poses interesting problems for any auditory system: In addition to simply detecting various frequencies, the animal must extract important information contained in each frequency channel of interest. Only recently have behavioral experiments demonstrated that an insect, the field cricket, actually makes use of frequency (Moiseff et al., 1978; Nolen and Hoy, 1986a). Because of its well-characterized acoustic behavior and its relatively simple auditory system, the field cricket provides an excellent system in which to address questions regarding the possible function and the physiological mechanism of frequency discrimination, in a behavioral context.

Flying crickets produce 3 distinct responses to sound, depending on the sound's temporal structure, intensity, and frequency content: Sounds at the carrier frequency of the species' calling song, 4-5 kHz are attractive as long as they are presented with an appropriate temporal pattern (Walker, 1957; Hill, 1974; Pollack 1982; Thorson et al., 1982; Stout et al., 1983; Pollack et al., 1984). Thus, phonotaxis to the calling song is optimized for characteristics of the male's song (see Huber, 1983). In contrast, ultrasound (frequencies between 20 and $100 \mathrm{kHz}$ ) evokes a "turning away" response (negative phonotaxis) at low to moderate intensities and an "evasive" response at high intensities (Moiseff et al., 1978; Nolen and Hoy, 1986a). These avoidance responses to high frequencies seem to be analogous to the antipredator, bat-avoidance behaviors of moths (Roeder, 1967).

Two classes of auditory interneurons in the cricket CNS ap- 
pear responsible for frequency discrimination. One group, including the neurons designated $\mathrm{ON} 1, \mathrm{ON} 2$, and $\mathrm{AN} 1$, is tuned to the species' calling song frequency of $4-5 \mathrm{kHz}$ and accurately codes temporal structure (Wohlers and Huber, 1982) and direction (Boyd and Lewis, 1983; Pollack, 1986). Other interneurons are sensitive to sounds over a broad range of frequencies, 3-100 kHz (Casaday and Hoy, 1977; Popov and Markovich, 1982; Moiseff and Hoy, 1983; Atkins and Pollack, 1986). For example, the identified prothoracic interneuron, Int-1, was first shown by Casaday and Ioy (1977) to respond during the interpulse intervals of the calling song, suggesting a possible role in social behavior. Later, Moiseff and Hoy (1983) showed that Int- 1 was also quite sensitive to ultrasonic frequencies up to at least $100 \mathrm{kHz}$; they therefore proposed that it functioned as a "bat-detector" and was important in the ultrasound avoidance behavior. Recently, we (Nolen and Hoy, 1984) tested the batdetector hypothesis directly by recording intracellularly from Int- 1 during performance of flight phonotaxis and found it to be crucial for ultrasound avoidance steering: Int-1 was both necessary and sufficient for the initiation of this behavior during the context of flight. However, it was sufficient only when it discharged action potentials above about 180-220 spikes/sec, the discharge rate produced by ultrasound that was of threshold intensity for the behavior.

Given Int-1's important role in eliciting avoidance steering and the observation that it responded to the calling song, it was surprising that the animal rarely performed avoidance steering in response to the calling song (Nolen and Hoy, 1986a). A critical response parameter of potential behavioral relevance is Int-l's high discharge rate in response to ultrasound. The purpose of this paper was to quantify Int-1's response over the range of frequencies encompassing the calling song and batlike ultrasound. We asked, Does Int-1 discharge at characteristically lower rates in response to calling song than to ultrasound? Intracellular recordings from Int-1 showed that it was indeed selective for ultrasound in terms of discharge rate. While it received excitatory postsynaptic potentials (EPSPs) in response to calling song, it also received quite strong inhibitory inputs as well (see also Casaday and Hoy, 1977; Moiseff and Hoy, 1983), which prevented it from responding much above 150 spikes/sec, too low to elicit avoidance steering. Thus, postsynaptic inhibition of Int-1 limits its response to the calling song and confers it with special response properties important for bat detection. Lowfrequency inhibition accounts for the tuning characteristics of the avoidance behavior, as well as the physiological and behavioral effects of 2-tone suppression (Moiseff and Hoy, 1983; Nolen and Hoy, 1986b). These results strengthen Int-1's proposed role in escape behavior; its poor and variable response to pulses of the calling song suggests a more limited role in social communication. These results have been reported previously in abstract form (Nolen and Hoy, 1982, 1983).

\section{Materials and Methods}

Animals. Adult female Teleogryllus oceanicus, 2-21 d postadult molt were obtained from our colony at Cornell. Care was taken during rearing to prevent a high density of nymphs, as this often resulted in loss of one or both forelegs, where the adult's ear is located. Even though nymphs regenerate the leg in subsequent molts, loss of the leg early in development has detrimental effects on the development of the ear (Hoy et al., 1978, 1985; Pallas and Hoy, 1986). Most animals were tested behaviorally for their response to 30 and $5 \mathrm{kHz}$ tone pulses in a flight phonotaxis assay (Nolen and Hoy, 1986a) before use in physiological experiments. Except where noted, animals exhibiting abnormal phonotaxis were not used. Behavioral and physiological tests were performed in a foam-lined, anechoic chamber that allowed acoustic stimulation from a set of speakers $0.7 \mathrm{~m}$ and $90^{\circ}$ on either side of the animals's longitudinal axis. These experiments were done under acoustic conditions similar to those of previous behavioral studies (Nolen and Hoy, 1984, 1986a, b).

Acoustic stimuli. Sound pulses were synthesized and delivered as described previously (Nolen, 1984; Nolen and Hoy, 1986a). Briefly, pure tones were produced by gating a sinewave generator with a trapezoidal envelope. Pulses $0.5-500 \mathrm{msec}$ were possible with carrier frequencies from 3 to $40 \mathrm{kHz}$. Unless otherwise indicated, a symmetrical rise and fall time of $5 \mathrm{msec}$ was used. Sound pressure levels were selectable by the use of a precision attenuator (HP350D) to within 1 decibel (dB) as measured with a Brüel and Kjær $1 / 4$ inch microphone (4135) and SPL meter (2203) at the location of the animal. The sound field was uniform to $\pm 1 \mathrm{~dB}$ within $3 \mathrm{~cm}$ of the preparation. The root-mean-square sound pressure levels are expressed in $\mathrm{dB}$ relative to $20 \mu \mathrm{Pa}$ (dB SPL). Except where noted, the maximum sound level achievable was at least $100 \mathrm{~dB}$ SPL.

The physiological identification of Int-1 required the use of 2-tone stimulation (sce Moiseff and Hoy, 1983). Two separate sound-generating systems were used. Tone pulses were gated synchronously, but their sound levels could be controlled independently by separate attenuators. The 2 signals were added together using unity-gain op-amp buffers bcfore powcr amplification and delivery from a wide-band speaker (Nolen and Hoy, 1986b). The spectral purity of synthesized acoustic stimuli, as determined with a real-time spectrum analyzer (Nicolet 444A) indicated harmonic distortion of less than $3 \%$ for pulses with carrier frequencies between 3 and $10 \mathrm{kHz}$ and less than $0.5 \%$ between 10 and $40 \mathrm{kHz}$.

Physiological recordings. Intracellular recording and staining was accomplished using microelectrodes filled with 3-5\% Lucifer yellow-CH $(100-200 \mathrm{M} \Omega)$ and conventional recording techniques. The animal was mounted ventral side up on a small platform and the forelegs waxed in flight posture using a low-melting-point wax (Kerr Sticky Wax); the large, posterior tympana faced away from the body (Moiseff and Hoy, 1983). A wax well was formed around the ventral part of the prothorax, which provided a reservoir for saline. The ventral cuticle was carefully disscetcd away, exposing the prothoracic ganglion. The wax reservoir was filled with saline (modified form of O'Shea and Adam's TES ringer; see Strausfeld et al., 1983) and changed periodically throughout the experiment. Care was taken not to damage the nerve roots and connectives nor the acoustic trachea between the 2 forelegs and thoracic spiracles; the trachea and spiracles are probably important in auditory function (Larsen and Michelsen, 1978; Wendler et al., 1980). Recordings were made from Int-1's axon in the cervical connectives and from its dendrites in the auditory neuropil of the prothoracic ganglion.

The ganglion was mechanically stabilized for recording by suspension on a wax-covered platform. An indifferent electrode ( $\mathrm{Ag}-\mathrm{AgCl}$ wire) was waxed into the saline reservoir anterior to the prothorax. The ganglionic sheath was treated briefly (2-4 min) with a small piece of pronasesoaked filter paper, followed by washout $(5 \times)$ with saline.

Physiological identification of Int -1 . Scarch stimuli consisted of 30 msec, $30 \mathrm{kHz}$ tone pulses at $60-70 \mathrm{~dB}$, repeated at $1 / 2 \mathrm{sec}$. Int-1 was putatively identified after intracellular penetration by 3 criteria: (1) A relatively high sensitivity to ipsilaterally presented ultrasound. (Here and throughout, ipsilateral refers to the side of the animal containing Int- 1 's axon and major dendrites; Int- 1 receives innervation from acoustic afferents from the ipsilateral ear. See Fig. 1.) (2) A short response latency to ultrasound $(10-15 \mathrm{msec}$ at $70 \mathrm{~dB})$ and longer latencies to tone pulses of the calling song ( $35 \mathrm{msec}$ at $70 \mathrm{~dB}$ ). (3) The presence of 2 -tone inhibition of the ultrasound response by the simultaneous presentation of a tone pulse of the calling song fundamental, $4-5 \mathrm{kHz}$ (Moiseff and Hoy, 1983; Nolen and Hoy, 1986b). Occasionally, the search procedure resulted in localization and subsequent penetration of the Omega neuron (Casaday and Hoy, 1977; now called ON1, using the terminology of Wohlers and Huber, 1982), which has some low-threshold sensitivity to ultrasound. The 3 test criteria reliably excluded $\mathrm{ON} 1$ and other neurons from putative identification as Int-1. In a number of cases we recorded and stained ultrasound-sensitive neurons that failed one or more of the criteria; in most cases, the neuron stained was $\mathrm{ON} 1$ and sometimes a calling song-sensitive ascending neuron (homologous to AN1 in Gryllus: Wohlers and Huber, 1978, 1982), which has a high threshold for ultrasound. Occasionally, an ultrasound-sensitive through- 


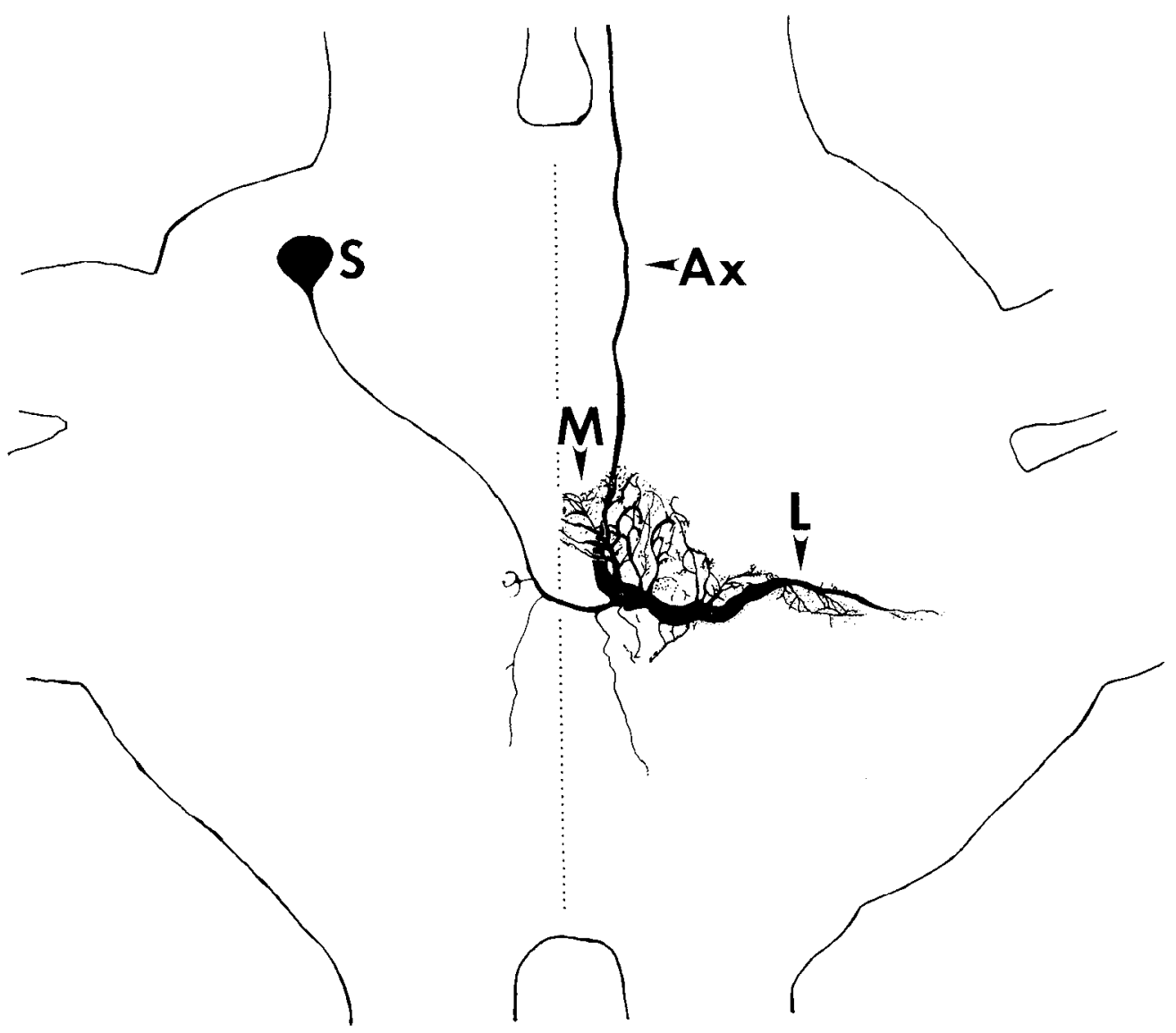

Figure 1. Drawing of an intracellularly stained Int-1, from a photographic reconstruction. $S$, soma; $A x$, axon; $M$, medial dendrites; $L$, lateral dendrite. Ventral view with anterior at the top of the page. The ganglion is approximately $800 \mu \mathrm{m}$ in diameter. Electrophysiological recordings were made from the region of the hemiganglion lateral to the midline (dotted line) and extending 1/4 of the width of the ganglion to about the location of the arrowhead labeled $L$. Recordings made in the lateral $1 / 4$ th of the ganglion were usually located near, or lateral to, the region of the lateral dendrite labeled $L$. The term ipsilateral refers to the side of the ganglion or animal containing Int-1's axon and major dendrites. Thus, all recordings reported here were ipsilateral with respect to Int1 's dendritic morphology. conducting fiber was stained; these had higher thresholds and longer latencies than Int-1 (Atkins and Pollack, 1986). A few Int-1 lype neurons also showed good sensitivity to $5 \mathrm{kHz}$; some, but not all, of these were correlated with abnormal Int-1 dendritic morphology and an abnormal appearance of the tympanal organ and with inconsistent behavior in phonotaxis assays (see below).

Morphological identification of Int-1. To ensure positive identification, cells putatively identified as Int-1 by their physiological properties were stained intracellularly with Lucifer yellow- $\mathrm{CH}$ (Stewart, 1978). The prothoracic ganglion was dissected out and fixed either in a phosphatebuffered formalin solution for $10 \mathrm{hr}$ or prefixed for $10 \mathrm{~min}$ in this solution and then fixed for $1 \mathrm{hr}$ in $6 \%$ formalin/methanol (Stewart, 1978). The tissue was then dehydrated in an ethanol series and cleared in methylsalicylate. Most ganglia were viewed as whole mounts under epifluorescence. Some were embedded in plastic (Ladd's ultra-low viscosity embedding medium) and sectioned at $30 \mu \mathrm{m}$ and then photographed. Int-1 was identified on the basis of its characteristic morphology (Moiseff and Hoy, 1983). Only physiological data collected from positively identified Int-1s are presented in this paper.

Quantification of response properties. Latency measurements, action potential counts, and discharge rate determinations were made with the aid of an analog-to-digital oscilloscope converter (Wavesaver, Epic Instruments), with a processing digital oscilloscope (Prowler, Norland Corp), or with a microcomputer using a purpose-built event counter. Initial and maximum instantaneous discharge rates were determined as the reciprocal time interval between action potentials and are expressed in spikes per second (sps). The initial insantaneous discharge rate was obtained from the first interspike interval, the maximum discharge rate from the shortest interspike interval of the response. The average discharge rate during the response was calculated as

$$
\frac{\text { (Number of action potentials) }-1}{\text { Duration of response }}
$$

and expressed in spikes per second (sps). Plots of instantaneous discharge rate versus time were obtained from the processing digital oscilloscope. Threshold tuning curves were obtained by determining the minimum sound level required to produce $3-4$ action potentials within $80 \mathrm{msec}$ of the stimulus tone pulse over a range of frequencies.

\section{Results}

\section{Int-1: an identified neuron}

Int- 1 was first identified in $T$. oceanicus by Casaday and Hoy (1977) and is probably homologous to AN2 in G. campestris and $G$. bimaculatus (Wohlers and Huber, 1978, 1982) and to similar neurons in a number of other field crickets (R. R. Hoy, unpublished observations). The characteristic L-shaped morphology of Int- 1 in $T$. oceanicus has been described (Moiseff and Hoy, 1983). Briefly, Int-1 is located in the prothoracic ganglion (Fig. 1) and receives innervation primarily from the afferents ipsilateral to its prominent lateral dendrite ( $L$, Fig. 1), which overlaps the afferent terminations in the auditory neuropil. The soma $(S)$ is located in the dorsal, anterior portion of the ganglion, contralateral to the axon $(A X)$ and the major dendrites. A large tuft of multibranched medial dendrites $(M)$ arises along the medial part of the lateral dendrite near the elbow of the $L$. Some of these medial dendrites project to the midline but stop short of projecting into the contralateral auditory neuropil. The axon arises at a right angle from the lateral dendrite and projects anteriorly through the ipsilateral cervical connective to the brain (Moiseff and Hoy, 1983).

In the course of 7 years work using several different histological methods, including intracellular dye injection as well as cobalt backfilling, workers in this laboratory have stained Int-1 in well over 1000 preparations. Recent work by others has suggested the existence of several L-shaped cells (Hutchings and Lewis, 1984); however, only 1 pair of identified neurons with 
A.

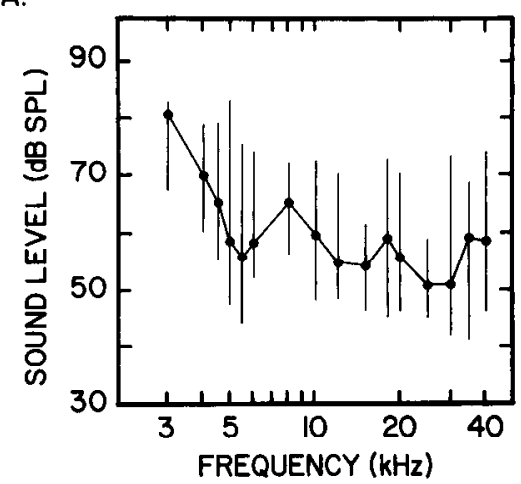

B.

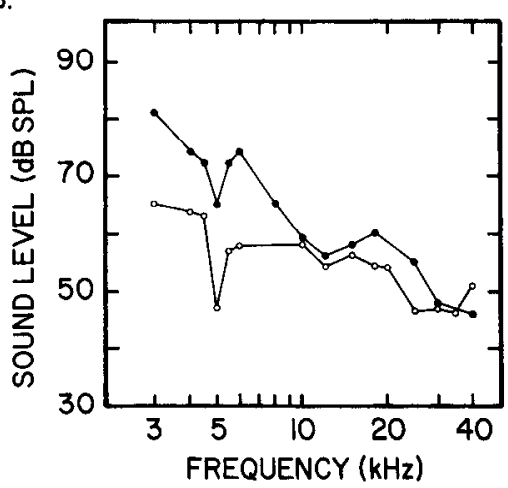

C.

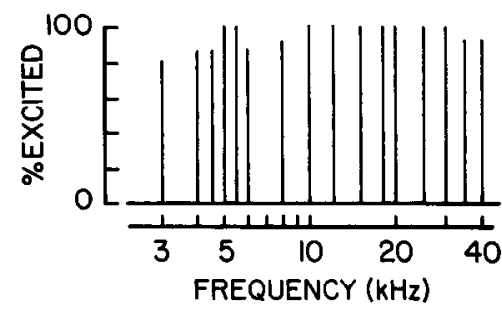

Figure 2. Frequency sensitivity of Int-1. The response of Int-1 to 30 Insec-long tone pulses ( $5 \mathrm{msec}$ rise and fall times) presented over the frequency range of $3-40 \mathrm{kHz}$. The speaker direction was $90^{\circ}$ to the left or right of the animal's longitudinal axis, toward the ear ipsilateral to Int-l's axon. $A$, Threshold excitatory tuning curve (see Materials and Methods) for Int-1 plotted as the median threshold sound level in $\mathrm{dB}$ (SPL). Error bars represent the range of thresholds at each frequency. From 8 positively identified Int- $1 \mathrm{~s}$. The ranges represent at least a $96.9 \%$ confidence interval for this sample size (Noether, 1971). B, Two excitatory tuning curves from Int-1s with large disparities in thresholds around the calling song frequency of $4-5 \mathrm{kHz}$. Note that both preparations were similarly sensitive to ultrasound. $C$, Percentage of Int-1s excited by single tone pulses up to a sound level of $80 \mathrm{~dB}$. In general, each preparation responded to tones over the entire range from 3 to $40 \mathrm{kHz}$ as long as the sound was loud enough. From 17 positively identified Int1s. (Because of technical limilations, not all were tested at each frequency, although each was tested over the majority of the range.) All percentages are significantly different from $50 \%$ (binomial test, $p<0.03$ ) for all frequencies (Noether, 1971).

the morphological properties attributed to Int-1 has been found, one on each side of the ganglion (Moiseff and Hoy, 1983). While the use of intracellular techniques does not discount the possibility of more than one class of L-shaped interneurons, the extensive database of backfill information supports only 1 morphological type of $\mathrm{L}$-cell in $T$. oceanicus. Int- 1 is readily distin- guishable from other identified auditory interneurons [e.g., the calling song pulse coding neuron, AN1 (Wohlers and Huber, 1982)] by its physiology (see Materials and Methods), as well as by its morphology.

Of a total of 96 preparations, $56 \mathrm{~L}$-shaped interneurons with the general Int-1 morphology were stained successfully by intracellular injection of Lucifer yellow. Fifty-three had the characteristic morphology and physiology of Int-1; 3 had the general morphological features attributed to Int-1 with the exception that the medial dendrites crossed the midline and terminated in the contralateral auditory neuropil. This was in striking contrast to the other $53 \mathrm{~L}$-shaped neurons stained with Lucifer yellow, in which the medial dendrites were restricted to the ipsilateral side of the ganglion. Most of these stains were very good, and many were excellent (very brightly stained); the 3 cells with crossover medial dendrites were stained no better than these. Failure to see crossover dendrites in the majority of the preparations was therefore not due to limitations of the Lucifer staining technique.

The morphology and physiology of the neurons with crossover medial dendrites were strikingly similar to those of animals that had been deafferented as nymphs by removal of the foreleg, where the ear develops (Hoy et al., 1978, 1985). It is known that carly deafferentation yiclds almost exclusively Int-1 type neurons with crossover medial dendrites, as seen in cobalt backfills (Timms intensified) and intracellular Lucifer yellow stains (Hoy et al., 1978, 1985; Pallas and Hoy, 1986). Int-1s with experimentally induced crossover dendrites, as well as the few found naturally in the colony, had unusually good responsiveness for calling song as well as for ultrasound. In addition, animals with crossover Int-1s had abnormal phonotactic behavior. While normal Int-1s showed some variation in response properties from preparation to preparation, especially for calling song (see below), the 3 crossover Int-1s encountered in this study were more extreme than expected for untreated preparations (T. G. Nolen, unpublished observations). Thus, the occurrence of crossover medial dendrites in Int-1 probably was the result of early deafferentation or damage to, and subsequent regeneration of, the leg. This could have happened during molting or as a result of injuries inflicted by conspecifics. This is supported by the observation that the incidence of abnormal morphology and phonotaxis behavior is dependent on high population densities of nymphs during rearing (T. G. Nolen and R. R. Hoy, unpublished observations). The data presented here are from the 53 Lucifer yellow-stained Int-1s with ipsilateral medial dendrites that came from animals with normal phonotactic behavior. We feel that these physiological data reflect the normal range of variability of Int-1.

\section{Frequency range of Int-1 response}

Moiseff and Hoy (1983) showed that Int-1 was excited by sounds from 8 to $100 \mathrm{kHz}$; we have concentrated on the range of sounds between 3 and $40 \mathrm{kHz}$, particularly around the fundamental frequency of the calling song of this species, 4-5 kHz. Int-1 received excitatory postsynaptic potentials (EPSPs) and discharged action potentials in response to sound pulses with carrier frequencies between 3 and $100 \mathrm{kHz}$, a range that encompasses the low-frequency sounds used by insects for social communication, as well as the ultrasonic frequencies used by a number of groups of insectivorous bats (Hoy et al., 1982). Representative threshold tuning curves for Int-l up to $40 \mathrm{kHz}$ are shown in Figure 2, $A$ and $B$. In Figure $2 A$ the median threshold 
A.

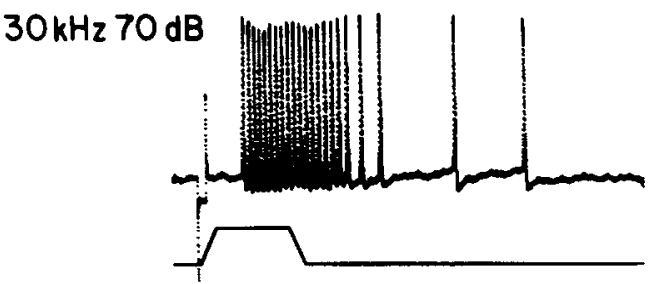

$5 \mathrm{kHz} 70 \mathrm{~dB}$

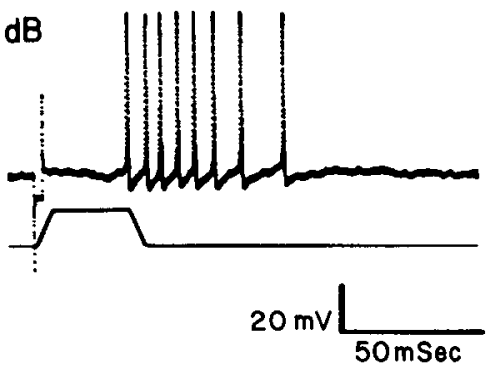

B.

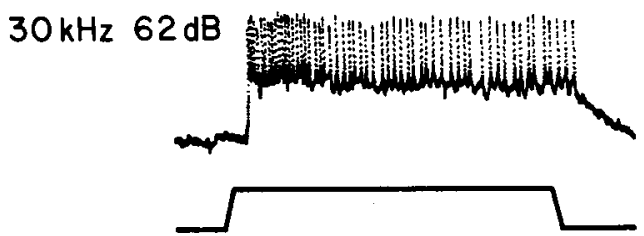

$5 \mathrm{kHz} 66 \mathrm{~dB}$

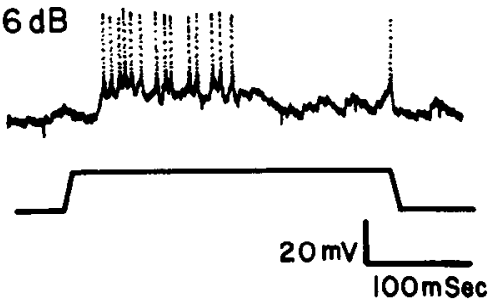

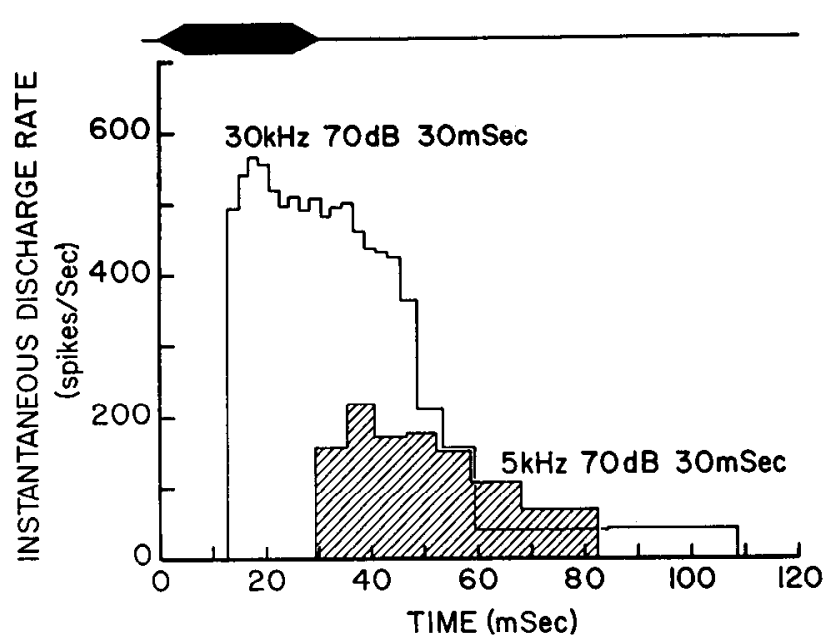

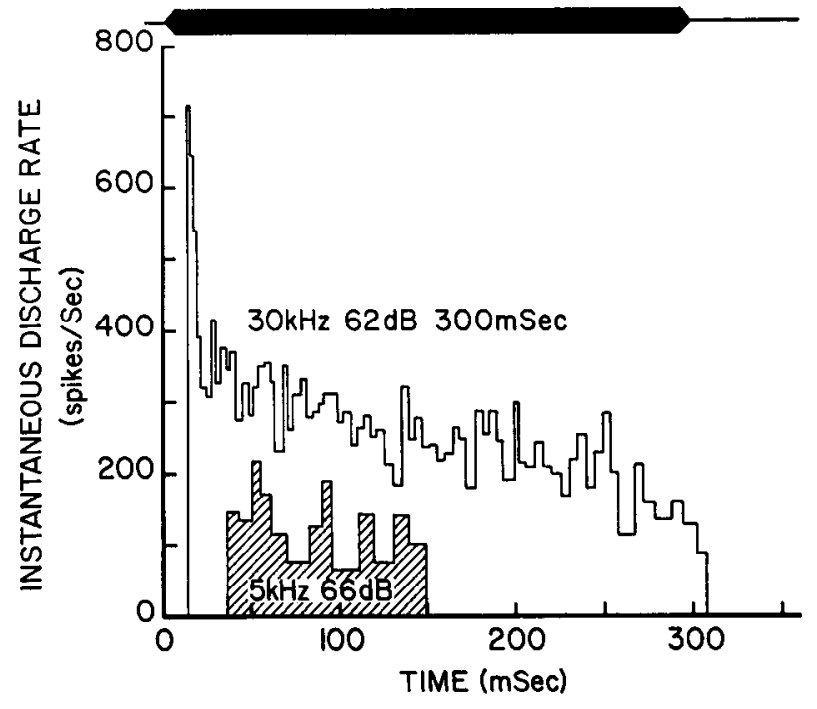

Figure 3. Comparison of Int-1's response to 30 and $5 \mathrm{kHz}$ tone pulses. Left, Intracellular records from Int-1. Right, Instantaneous discharge rate versus time in response to the tone pulse. The tone began at $0 \mathrm{msec}$. All stimuli presented to the ipsilateral ear. $A$, Response to short tone pulses ( $30 \mathrm{msec}$ duration, $5 \mathrm{msec}$ rise and fall times) as recorded in the axon. Note the relatively low discharge rate and longer latency of the $5 \mathrm{kHz}$ response compared to that for $30 \mathrm{kHz}$. $B$, Response to longer tone pulses ( $300 \mathrm{msec}, 5 \mathrm{msec}$ rise and fall times) as recorded in the dendritic region (probably a medial dendrite, see text).

sound level for excitation (see Materials and Methods) is plotted along with the range of thresholds for 8 positively identified Int$1 \mathrm{~s}$. There was a small region of low-threshold sensitivity near $5 \mathrm{kHz}$ and a broad range of low-threshold sensitivity at frequencies greater than $8 \mathrm{kHz}$. Most of the cells tested responded over the entire range, as long as the tone pulse was loud enough (Fig. $2 C$ ). However, there was quite a bit of variability in the thresholds over this range of frequencies, especially near the calling song frequency (see Fig. $2 A$ ). Figure $2 B$ shows excitatory tuning curves for 2 Int- $1 \mathrm{~s}$ with the largest observed disparities in shape; most of the variability was centered around $5 \mathrm{kHz}$, the calling song frequency.

$\Lambda$ s shown in Figure 2, simple threshold excitatory tuning curves tend to suggest that Int- 1 is often just as sensitive to the calling song as it is to ultrasound. However, threshold tuning curves do not show how well the cell responds above threshold over this range of frequencies. In fact, the response to frequencies greater than $8 \mathrm{kHz}$ was qualitatively and quantitatively different from that to lower frequencies. A major distinguishing characteristic of Int-1 is its relatively strong response to ultrasound, compared to its response to tone pulses of the calling song. Figure 3 (left panel) shows intracellular records from Int- 1 in response to tone pulses of 5 and $30 \mathrm{kHz}$. Note that while Int-1 was excited to fire action potentials by $5 \mathrm{kHz}$ tone pulses, its response was much less vigorous than that to $30 \mathrm{kHz}$ tone pulses (e.g., Fig. $3 A$ ), especially in terms of the number of action potentials produced during the burst, the discharge rate and the latency of its response (Fig. 3, $A$ and $B$, right panel).

In general, Int-1 produced fewer action potentials, lower discharge rates, and longer latencies in response to tone pulses of lower carrier frequencies. Figure $4, A$ and $B$, shows examples of the number of action potentials discharged and the response latency for tone pulses of various frequencies and sound levels (isointensity curves). The greatest number of action potentials 
A.

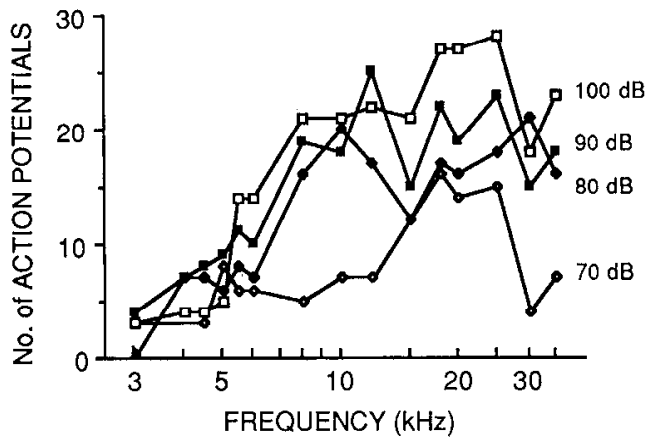

B.

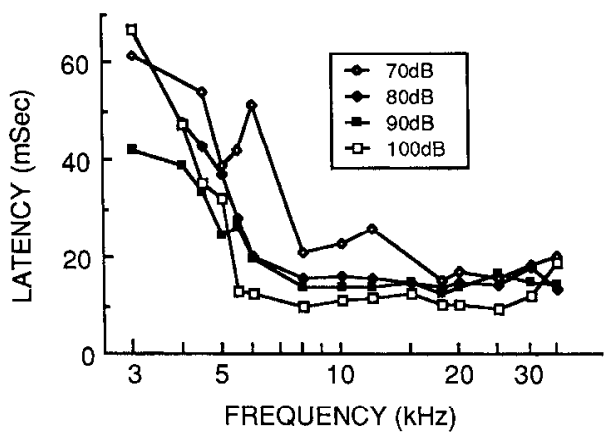

C.

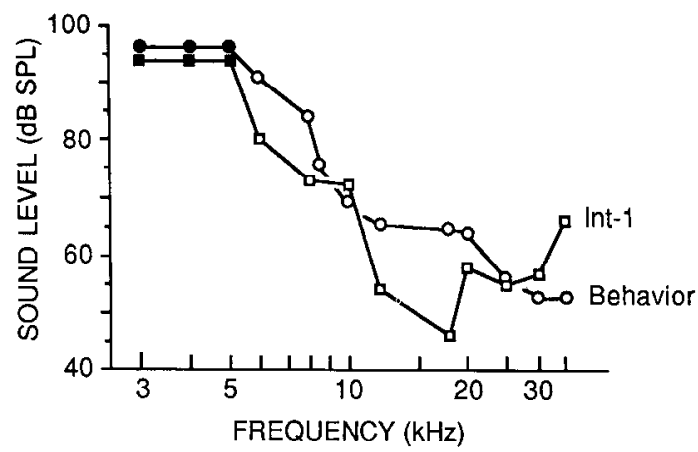

Figure 4. Isointensity, latency, and isorate curves for Int-1. A, Isointensity: Typical example of the number of action potentials elicited in Int-1 in response to $30 \mathrm{msec}$ tone pulses ( $5 \mathrm{msec}$ rise and fall times) over a range of carrier frequencies and sound levels. Similar results were obtained in 10 positively identified Int-1 s. $B$, Latency: Response latency for the same preparation as in $A$. Latency was measured from the arrival of the acoustic stimulus at the preparation to the first action potential in Int-1. C, Comparison of the isorate tuning curve of Int-1 and the behavioral tuning curve for avoidance steering. Squares, Example of the sound level required to produce an average discharge rate in Int-1 of 150-180 sps (30 msec tone pulses). Circles, Threshold tuning curve for negative phonotactic steering behavior (from Nolen and Hoy, 1986a). Solid symhols indicate the failure to reach these behavioral or physiological criteria at the sound levels indicated.

(Fig. 4A), the lowest thresholds (see Fig. 2A), and the shortest latencies (Fig. $4 B$ ) occurred in response to high-frequency tone pulses. Moreover, the sound level required to cause Int- 1 to discharge above 150-180 sps was greater for frequencies near the calling song than for higher frequencies (Fig. 4C). While the shape of this isorate tuning curve for this criterion response was very similar to the tuning curve for the avoidance behavior (Fig. $4 C$ ), it was quite different from the threshold tuning curve for
A.

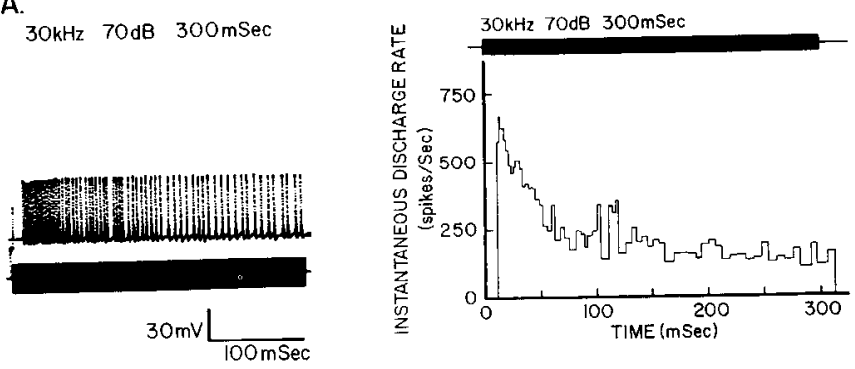

B.

$30 \mathrm{kHz} 70 \mathrm{~dB} \quad 30 \mathrm{mSec}$
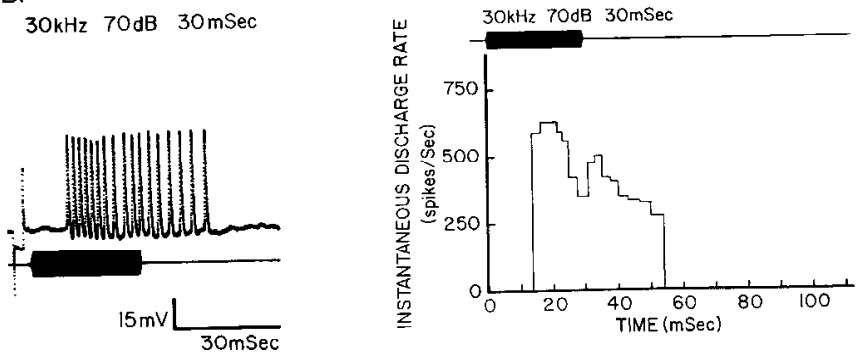

C.

$30 \mathrm{kHz} 70 \mathrm{~dB} 8 \mathrm{mSec}$
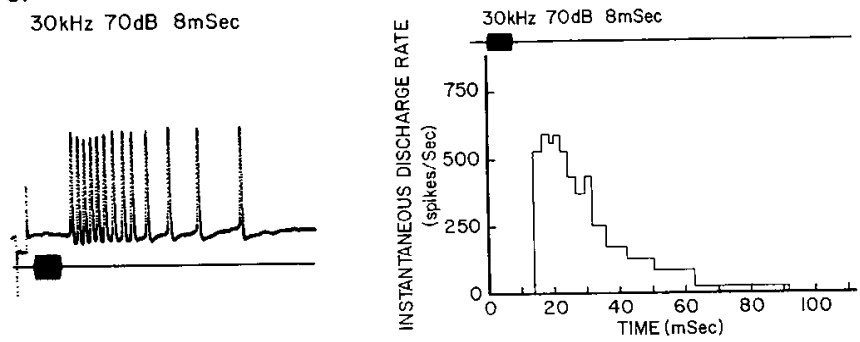

D.

$30 \mathrm{kHz} \quad 70 \mathrm{~dB} \quad 0.5 \mathrm{mSec}$
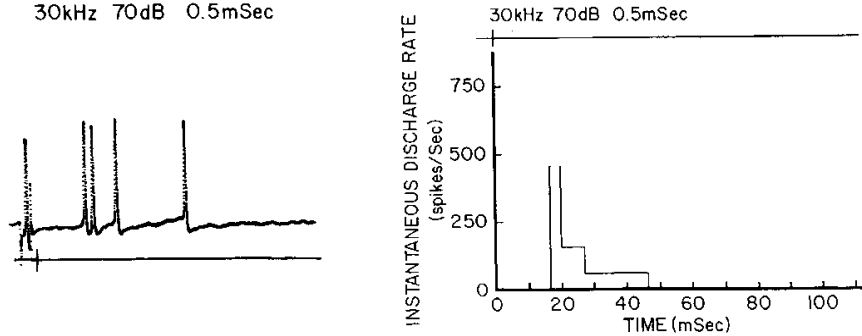

E.

$30 \mathrm{kHz} \quad 80 \mathrm{~dB} \quad 0.5 \mathrm{mSec}$
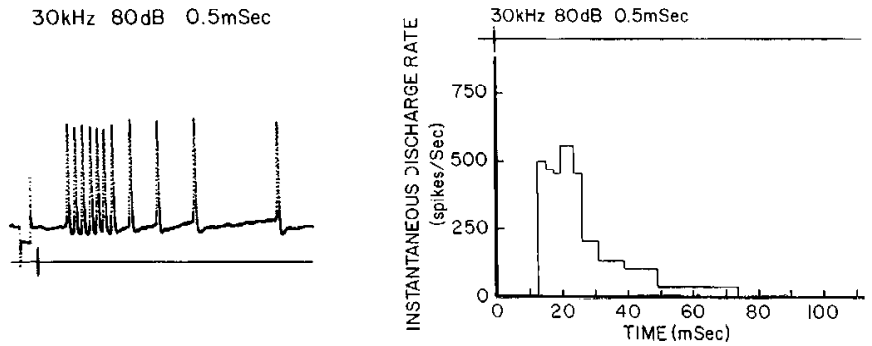

Figure 5. Response of Int-1 to varying duration pulses of $30 \mathrm{kHz}(250$ $\mu \mathrm{sec}$ rise and fall times), presented ipsilaterally. Axonal recordings. Calibration (in $B$ ) is for $B-E$. 
A. $30 \mathrm{kHz} 68 \mathrm{~dB}$

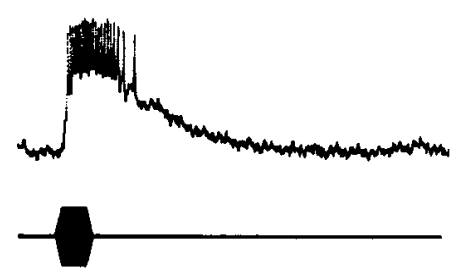

$$
10 \mathrm{mvL}
$$

C. $40 \mathrm{kHz} 83 \mathrm{~dB}$

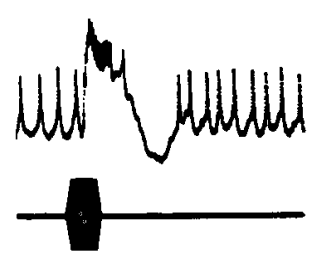

$$
10 \mathrm{mVL}
$$

B. $30 \mathrm{kHz} 80 \mathrm{~dB}$

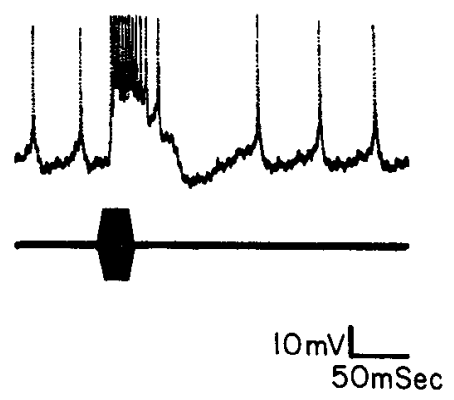

D. $30 \mathrm{kHz} 70 \mathrm{~dB}$

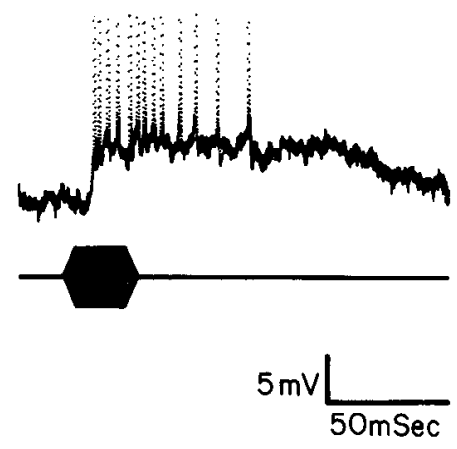

Figure 6. Examples of PSPs recorded intracellularly from Int-1. Recording sites were in the auditory neuropil. $A$, Medial recording site; large EPSP with nonovershooting action potentials riding on top. $B$, Medial recording site from another preparation. Note that the action potentials were relatively large, as was the EPSP; note also the large IPSP following stimulation. The recording in $A$ was probably from one of the smaller medial dendrites (see Fig. 1, $M$ ), whereas that in $B$ was probably from the larger, proximal portion of the lateral dendrite near the elbow of the $L$, or from the integrating segment (the ascending portion of the cell, anterior to the lateral dendrite). $C$. Recording from the lateral $1 / 4$ th of the ganglion. Note the small action potentials riding on the relatively large EPSP and the large poststimulus IPSP. Because of the electrode position, this recording must have been from a latcral process of the cell, possibly the large lateral dendrite. $D$, Axonal recording. Note the high gain (the action potentials are clipped).
Int-1 (compare Figs. $4 C$ and $2 B$ ), an observation indicative of considerable integration of frequency information at the level of a primary sensory interneuron.

\section{Pattern of discharge in response to sound \\ Batlike ultrasound}

Bats produce echolocation vocalizations from $10 \mathrm{kHz}$ to greater than $120 \mathrm{kHz}$ (Novick, 1977). Int-1 typically responded to sounds over this range with a very rapid burst of action potentials (Fig. $3, A$ and $B$ ); initial instantaneous discharge rates were as high as 800 sps at high intensities (Figs. 3 and 10C). Usually, the discharge rate rose rapidly, with the maximum instantaneous discharge rate occurring within the second or third interspike interval. The initial high rate portion of the burst lasted 5-20 msec, depending on the sound intensity and pulse duration (Figs. 3 and 5). At longer pulse durations, the discharge rate dropped to roughly a third of the initial rate after $30-50 \mathrm{msec}$ and remained there for the duration of the stimulus pulse (Fig. $5 \mathrm{~A}$ ).

Bats produce ultrasonic pulses of varying durations, but short$\mathrm{cn}$ them to as little as $0.5 \mathrm{msec}$ when approaching a target (Griffin et al., 1965; Simmons and Kick, 1983). As shown in Figure 5, the duration of the action potential burst in Int- 1 decreased as the tone pulse was shortened but the initial spike rate remained unchanged until pulse durations were less than or equal to 0.5 msec (Fig. 5, $A-D$ ). Nevertheless, Int-1 responded well to very short pulses of ultrasound; for example, a $0.5 \mathrm{msec}$ pulse of 30 $\mathrm{kHz}$ at $80 \mathrm{~dB}$ produced a burst of about 11 action potentials, similar in form to that produced by longer pulses (compare Fig. $5, E$ and $C$ ), except that the discharge rate dropped more rapidly after the first $15 \mathrm{msec}$ of response (Fig. $5 E$ ). In addition to shorter ultrasonic pulses, an insect being pursued by a bat would hear louder pulses as the bat approached closer (Simmons and
Kick, 1983). Int-1's reduced response to short ultrasonic pulses could be compensated for by increasing the sound level by 10 $\mathrm{dB}$, as in Figure $5 E .{ }^{1}$

\section{Calling song}

The response to low-frequency tone pulses was strikingly different from that to ultrasound; pulses below $8 \mathrm{kHz}$ rarely caused Int-1 to discharge above $300 \mathrm{sps}$ initially and above $150 \mathrm{sps}$ averaged over the burst (compare its response to $30 \mathrm{kHz}$ : Figs. 3,4 , and 10 ). The discharge rate rose more slowly in response to 5 than to $30 \mathrm{kHz}$ (Fig. 3, $A$ and $B$, right-hand panel), with maximum instantaneous rates usually reaching no higher than 240 sps (see also Fig. 10).

\section{Synaptic inputs \\ Excitation}

Large compound PSPs could be recorded in a variety of neuropilar sites in Int-1. Since Lucifer yellow diffuses rapidly throughout the cell, the exact location of the recording site could not be determined with certainty following dye injection. However, based on general electrode placement in the ganglion (i.e., medial or lateral) and, occasionally, on fortuitous Lucifer stains in which the electrode track was visible, and on assumptions regarding the passive conduction of action potentials and synaptic potentials, it is possible to suggest sites of synaptic inputs onto Int-1.

Generally, the form and relative amplitude of PSPs and action

'Bat sonar can also include frequency modulation (e.g., rapid sweeps in carrier frequency), as well as various harmonic components (Novick, 1977). Preliminary experiments (T. G. Nolen, unpublished observations) have shown that Int-1 is sensitive to these complex sounds (also see Popov and Markovich, 1982), but a systematic study was not undertaken. 
potentials depended on electrode placement. Some recordings in the medial $1 / 8$ to $1 / 4$ of the ganglion revealed very large EPSPs (up to $40 \mathrm{mV}$, the largest recorded) in response to ultrasound, with nonovershooting action potentials riding on top (Fig. 6A); also, multiple, successive penetrations of the same cell could be made while recording in this region of the ganglion (as judged by staining attempts) by driving the microelectrode through about $30 \mu \mathrm{m}$ of neuropil at a depth of about $200 \mu \mathrm{m}$ from the ventral surface. These observations suggest that it is likely that these recordings were made in the fine medial dendrites that branch profusely in this region of the auditory neuropil (Fig. $1 M$ ). In other medial recordings, large overshooting (or nearly overshooting) action potentials, relatively large EPSPs, and large IPSPs following the EPSPs at the end of an ultrasound pulse were observed (Fig. 6B). These recordings were probably from the integrating region or the proximal portion of one of the medial dendrites or from the medial portion of the large lateral dendrite (see Fig. 1).

Recordings from the lateral quarter of the ganglion revealed large EPSPs and IPSPs and small electrotonically conducted action potentials riding on the EPSPs (Fig. $6 \mathrm{C}$ ); the lateral position of the electrode suggests that these recordings were made from the large lateral dendrite or from a proximal portion of a small dendritic branch of the lateral dendrite. We also recorded PSPs in the axon as it left the ganglion and ascended the cervical connective (Fig. 6D). These PSPs were of extremely low amplitude but were nevertheless visible at high gain.

There was considerable variation in the amount of spontaneous activity recorded in Int- 1 . For example, most cells showed a low level of apparently random (nonperiodic) discharge, at rates less than 20 sps. In these cases, action potentials rose from unitary EPSPs (these can be seen in Fig. $7 A$, in the $300 \mathrm{msec}$ before stimulation, and in Fig. 6, $B$ and $C$ ); the cell was usually silent for a short time following stimulation, suggesting strong poststimulus inhibition (e.g., Fig. 6, $B$ and $C$ ). In some preparations, while the action potentials were of reasonable amplitude, the cell had a tonic level of discharge $>60 \mathrm{sps}$ (Fig. 6C), suggesting that it may have been depolarized slightly during penetration. However, such rates of spontaneous discharge were not necessarily associated with possible damage due to penetration: Similar rates were observed in extracellular recordings of putatively identified Int-1s as seen in the anterior cervical connectives using suction electrodes (Moiseff and Hoy, 1983; T. G. Nolen, unpublished observations). The occurrence of hyperpolarizing PSPs (IPSPs, see below) was not correlated with a tonic discharge level of this magnitude and hence on the cell being depolarized.

The phasic/tonic discharge of Int-1 was reflected in the shape of the compound EPSPs recorded in the dendrites and integrating segment. The compound EPSP produced upon ultrasonic stimulation rose very rapidly to peak amplitude within 5 msec and then decreased slightly over 5-20 msec to a level that lasted the duration of the tone pulse (Figs. $3 B$ and 6). The rapid rise accounted for the short latency of action potential generation and for the high instantaneous discharge rates. The tonic plateau level of the EPSP accounted for the sustained, lower discharge rate during long tone pulse stimulation (Fig. $3 B$ ). Some of the reduction in amplitude of the later part of the EPSP (Fig. 6) may be the result of strong contralateral inhibition as described by Moiseff (1980; see also Moiseff and Hoy, 1983), which was seen as an IPSP following the end of the sound pulse (for ex- ample, see Fig. 6, $B$ and $C$ ); this late-acting IPSP seems to account for suppression of the background discharge rate following acoustic stimulation (Fig. 6, $B$ and $C$; see also Moiseff and Hoy, 1983). [Monaural stimulation (T. G. Nolen, unpublished observations) suggests that somc of the reduction of EPSP amplitude could be due to adaptation of the afferent response.] The late, poststimulus IPSP was similar to those seen in other cricket auditory interneurons following tone pulse stimulation, for example, AN1 and ON1 in T. oceanicus (T. G. Nolen, unpublished observations) and in Gryllus bimaculatus and $G$. campestris (Wohlers and Huber, 1982). The late-acting IPSP was of contralateral origin, as shown by ipsilateral deafferentation (Fig. $8 A)^{2}$; contralateral inhibition is produced over the entire range of frequencies tested, 3-40 kHz (Moiseff and Hoy, 1983; T. G. Nolen, unpublished observations).

\section{Low-frequency postsynaptic inhibition}

Much of the difference in response to high and low frequencies could be attributed to another, ipsilateral source of postsynaptic inhibition of short latency and tuned to low frequencies (Fig. 7). Low-frequency pulses near the calling song fundamental elicited an IPSP as well as an EPSP (Fig. 7B). This IPSP was of short latency (10 msec minimum) and effectively shunted the rise of the EPSP, preventing the cell from rapidly reaching threshold (Fig. 7A). It was mediated ipsilaterally, as was the EPSP (Fig. $8 B$ ), and was thus distinct from that mediating contralateral inhibition (Moiseff and Hoy, 1983). The short-latency, ipsilateral IPSP could be seen in the integrating segment (Fig. $7, A$ and $C$ ), in the lateral dendrite (Fig. $8 B$ ), and sometimes in the proximal part of the axon (Fig. $7 B$ ) but only with difficulty in the distal region of the medial dendrites (Fig. 9). Current injection could reveal it in the medial dendrites (Fig. 9), suggesting that the source of synaptic current was electrically distant from the distal medial dendrites, although not too distant since the sign of the IPSP could be reversed by injected current (see $-4 \mathrm{nA}$ in Fig. 9). A short-latency, ipsilateral IPSP was never observed for high-frequency tone pulses, above $10 \mathrm{kHz}$.

\section{Dynamic range of response}

Int-1 operates over a characteristically wide range of ultrasonic intensities, from 45 to $100 \mathrm{~dB}$ (Fig. 10). The number of action potentials per stimulus increased monotonically over this range (Fig. 10A). The average discharge rate per burst increased until about 85-90 dB, where it startcd to plateau (Fig. 10B) even though the number of action potentials, as well as the burst duration (not shown), continued to increase at higher sound levels. The threshold sound level for negative phonotactic steering in response to $30 \mathrm{kHz}$ pulses was about $58 \mathrm{~dB}$ for the animals used in Figure 10 (arrowhead); the average discharge rate in Int- 1 at this sound level was about $180 \mathrm{sps}$, in agreement with our previous observations (Nolen and Hoy, 1984).

In contrast, the response range for $5 \mathrm{kHz}$ pulses was much smaller (Fig. 10, $A$ and $B$ ). The number of action potentials, the average discharge rate, and even the initial instantaneous discharge rate all demonstrated a distinct nonmonotonic relation-

\footnotetext{
${ }^{2}$ Figure $8 a$ shows a recording from the axon as it leaves the ganglion, several hundred microns from the dendrites (see Fig. 1). The small amplitude of this IPSP is not because the inhibition is weak, but because of electrotonic decrement between the locus of this synaptic input (dendrite) and the recording site (the axon). The fact that this PSP can be seen in the axon at all is consistent with the fact that it is functionally quite strong.
} 
A.

\section{$5 \mathrm{kHz} 80 \mathrm{~dB}$}
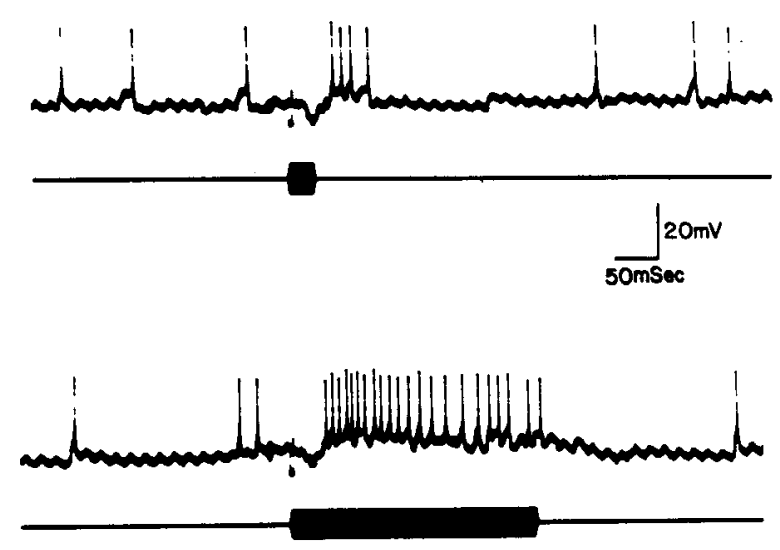

B.

\section{$5 \mathrm{kHz} 90 \mathrm{~dB}$}

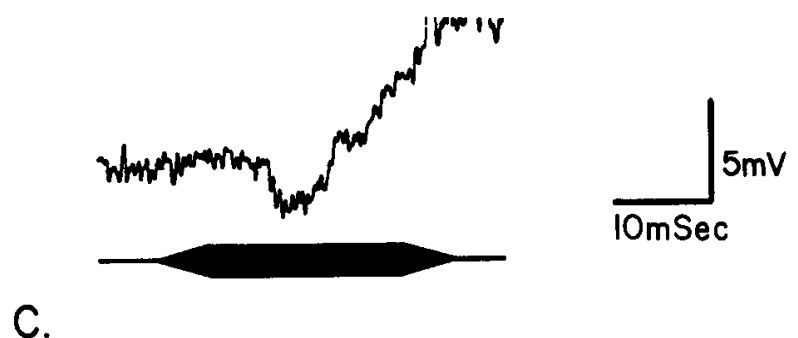

$5 \mathrm{kHz} 7 \mathrm{ddB}$
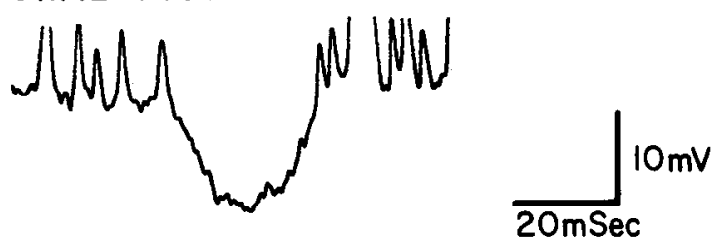

Figure 7. Response of Int-1 to $5 \mathrm{kHz}$ tone pulses as recorded in the medial $1 / 4$ th of the ganglion $(A, C)$ and from the axon $(B)$. The $5 \mathrm{kHz}$ tone pulses elicted a short-latency IPSP followed by an EPSP, which often resulted in action potential generation, with a longer latency than for $30 \mathrm{kHz}$ stimulation. (In $B$, the action potentials are off scale because of the high gain.) The records in $B$ and $C$ were signal-averaged 5 and 7 times, respectively. Note that in $C$ the averaged and summed action potentials before and after the IPSP were not synchronized with the stimulus pulse and were therefore reduced in amplitude during the averaging process. No action potentials were produced during the initial $20 \mathrm{msec}$ of the IPSP.

ship, increasing up to about $65 \mathrm{~dB}$, where they plateaued, and then decreasing above $90 \mathrm{~dB}$. This suggests that for low frequencies, inhibition at higher intensities reduced the effectiveness of the excitatory synaptic inputs. Several lines of evidence suggest that this source of low-frequency inhibition at high intensities was the short latency, ipsilateral IPSP rather than the broadly tuned contralateral IPSP. First, the initial and maximum discharge rates, which are sensitive measures of the effect of early, short-latency inhibition (Figs. 7, 8) decreased for high- intensity tone pulses of $5 \mathrm{kHz}$ but not for $30 \mathrm{kHz}$ pulses (Fig. $10 \mathrm{C}$ ). For example, initial discharge rates in response to $30 \mathrm{kHz}$ tones increased 8 -fold to nearly 800 sps over the range $45-100$ $\mathrm{dB}$. In contrast, initial discharge rates for $5 \mathrm{kHz}$ tones increased to a peak of only 200 sps by $75 \mathrm{~dB}$ but then dropped at higher sound levels. Second, preliminary experiments using closedficld (monaural) stimulation, as well as experiments following contralateral deafferentation, produced stimulus-response curves for 5 and $30 \mathrm{kHz}$ stimulation similar to those shown here in
A.

$30 \mathrm{kHz} 100 \mathrm{~dB}$
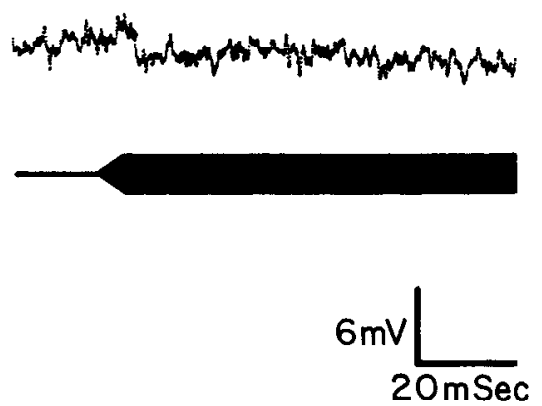

B.

\section{$5 \mathrm{kHz} 80 \mathrm{~dB}$}
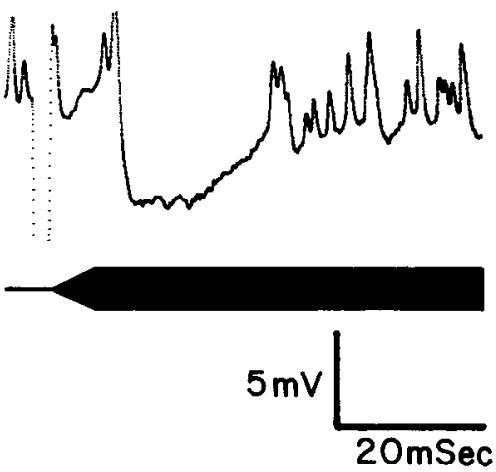

Figure 8. The source of PSPs in Int-1. A, Axonal recording following removal of the ear ipsilateral to the axon. Stimulation with loud $30 \mathrm{kHz}$ tone pulses to the intact side shows a small amplitude IPSP (small because of the distance between the dendrites and the axonal recording site), which appears to be responsible for contralateral inhibition (Moiseff and Hoy, 1983); the EPSP is gone (compare with Fig. $6 D$, the same preparation). $B$, Recording from the lateral $1 / 4$ th of the ganglion following removal of the ear contralateral to the axon. Stimulation with $5 \mathrm{kHz}$ tone pulses revealed a strong, short-latency IPSP, followed by an EPSP. Thus, excitation is mediated by the ipsilateral ear and inhibition can be elicited by both ears (Moiseff and Hoy, 1983). This record was averaged 7 times. 


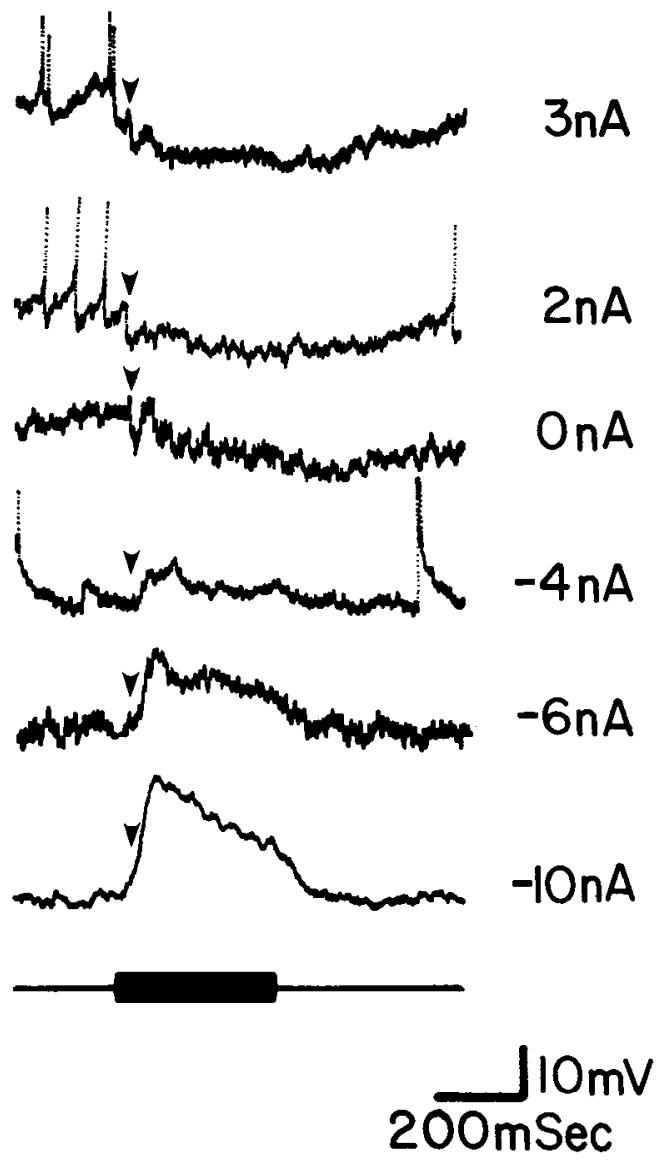

Figure 9. The short-latency, $5 \mathrm{kHz}$ IPSP is a conductance increase PSP, as shown by current injection. Current was injected into Int-1 in a medial dendrite. Injected current shown in nanoamperes $(\mathrm{nA})$ on the right. A low-intensity $5 \mathrm{kHz}$ tone pulse $(50 \mathrm{~dB}, 300 \mathrm{msec}$ duration, 5 msec rise and fall time) produced a small amplitude IPSP (arrowhead) followed by a small EPSP (see $0 \mathrm{nA}$ of injected current). A later, poststimulus IPSP can also be seen. Often, in medial recordings such as this, the EPSPs were of large amplitude while the action potentials were small; both the ipsi- and contralateral IPSPs were of small amplitude, suggesting that they were produced at a site electrically distant from the recording. Current injection could sometimes reveal an underlying IPSP. Depolarizing current caused an increase in amplitude of the IPSP and a reduction of the amplitude of the EPSP; hyperpolarizing current ( -4 to $-10 \mathrm{nA}$ ) caused the IPSP to reverse at levels just below the resting potential (see $-4 \mathrm{nA}$ ) while the EPSP increased in amplitude. Because of increased noise of the Lucifer electrodes during current injection, the rccordings for $3 \mathrm{nA}$ and for $-10 \mathrm{nA}$ were averaged 3 and 8 times, respectively.

which both the ipsi- and contralateral pathways were intact ( $T$. G. Nolen, unpublished observations).

At higher intensities ( $>90 \mathrm{~dB}$ ), the response to $5 \mathrm{kHz}$ could be put into 2 categories: (1) about half the preparations were nearly completely inhibited (Fig. 11A), whereas (2) the other half showed a decline in response from 70 to $95 \mathrm{~dB}$, where they abruptly increased their firing rates as the sound level exceeded $100 \mathrm{~dB}$ (Fig. $11 \mathrm{~B}$ ). In the first case, while the number of action potentials decreased at high sound levels, a short-latency action potential (at about $15 \mathrm{msec}$ ) was usually elicited, apparently "breaking through" the inhibition at sound levels above $80 \mathrm{~dB}$ (see Fig. 11A, 80-100 dB). In the latter case, the short-latency, "breakthrough" action potential was immediately followed by a large burst of action potentials (see Fig. 11B, 90-100 dB; also see Fig. 10, for the 2 classes at high sound levels). In these cases, the initial discharge rates were high (Fig. 10C) and the latencies short, sometimes approaching those of loud ultrasound (Fig. $10 D$ ). Thus, excitation could sometimes overcome the low-frequency inhibition. Breakthrough excitation suggests that, while the strength of both excitation and inhibition increased as the intensity of the stimulus increased, only at the very highest intensities $(>100 \mathrm{~dB})$ was the strength of inhibition unable to prevent strong excitation; otherwise, inhibition usually dominated.

The latency for action potential generation was shorter for 30 $\mathrm{kHz}$ pulses than for $5 \mathrm{kHz}$ pulses (Fig. 10D). For $30 \mathrm{kHz}$, latencies dropped about 20 msec over the range of 55-85 dB to less than $10 \mathrm{msec}$. The latencies for $5 \mathrm{kHz}$ pulses dropped to $17 \mathrm{msec}$ by about $90 \mathrm{~dB}$; above $90 \mathrm{~dB}$, some cells showed increased latencies, while most (those showing breakthrough excitation) had latencies as short as those for ultrasonic stimulation.

\section{Response to trains of pulses}

Although Int-1 received EPSPs in response to tone pulses at the calling song frequency, it did not follow trains of $5 \mathrm{kHz}$ tone pulses very well; its response decremented substantially (Fig. $12 B$ ) to successive pulses at low to moderate sound levels (70 $\mathrm{dB}$ was the "best" sound level for $5 \mathrm{kHz}$ (Fig. 10)), suggesting that the effects of low frequency inhibition built up over successive stimulations, although adaptation of the $5 \mathrm{kHz}$ afferents could also be important. In addition, Int-1's response to trains of calling song pulses was quite variable from preparation to preparation, as was "breakthrough" excitation. [See Wohlers and Huber (1982) for similar observations of the homologous neuron AN2 in Gryllus.] However, Int-1 did reliably follow trains of ultrasonic pulses, even pulses of very short duration (Fig. 12, $A$ and $C$ ). Thus, failure to follow model calling song was not due merely to adaptation of the sensory afferents or to fatigue of the afferent-Int-1 synapses but probably to low-frequency inhibition. Failure to follow model calling song trains makes it doubtful that it could accurately code the calling song's temporal properties or direction.

\section{Discussion}

Functional role of Int-1 in behavior

Int-l's response to various acoustic stimuli have suggested at least 2 roles in behavior, neither being necessarily mutually exclusive. (1) Int-1 responds to the calling song fundamental and could therefore be important during social interactions (Casaday and Hoy, 1977; Wohlers and Huber, 1978; Popov and Markovich, 1982; Hutchings and Lewis, 1984). (2) Int-1's great sensitivity to ultrasound has implicated it as a "bat detector" (Moiseff, 1980; Popov and Markovich, 1982; Moiseff and Hoy, 1983). Support for the bat-detector hypothesis has been provided by observations of the animal's phonotactic behavior in response to batlike ultrasound and by direct functional tests (necessity and sufficiency) of Int-1's role in flight behavior (Nolen, 1984; Nolen and Hoy, 1984, 1986a, b). To further investigate Int-1's role in behavior, we have examined in detail its response to 2 likely sources of environmental sounds, batlike ultrasound and cricket calling song.

\section{Postsynaptic inhibition}

Postsynaptic inhibition plays an important role in shaping Int1 's response to sound. The results presented here clearly show that acoustic stimulation produces both excitatory and inhibi- 
A.

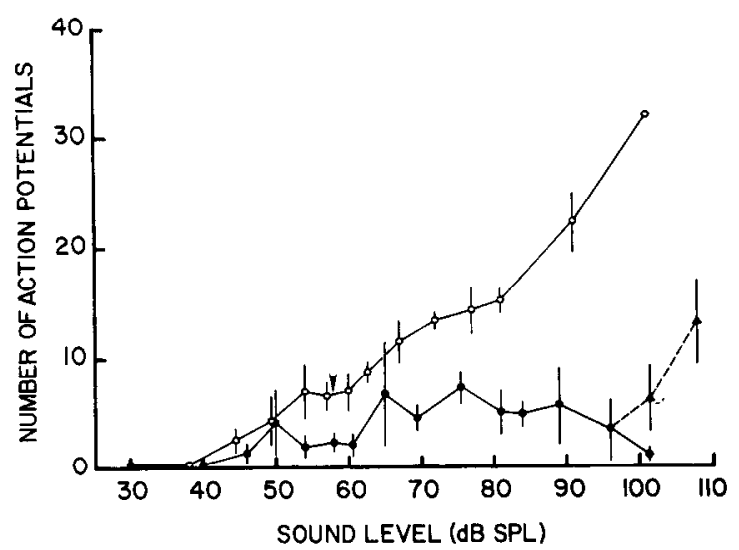

B.

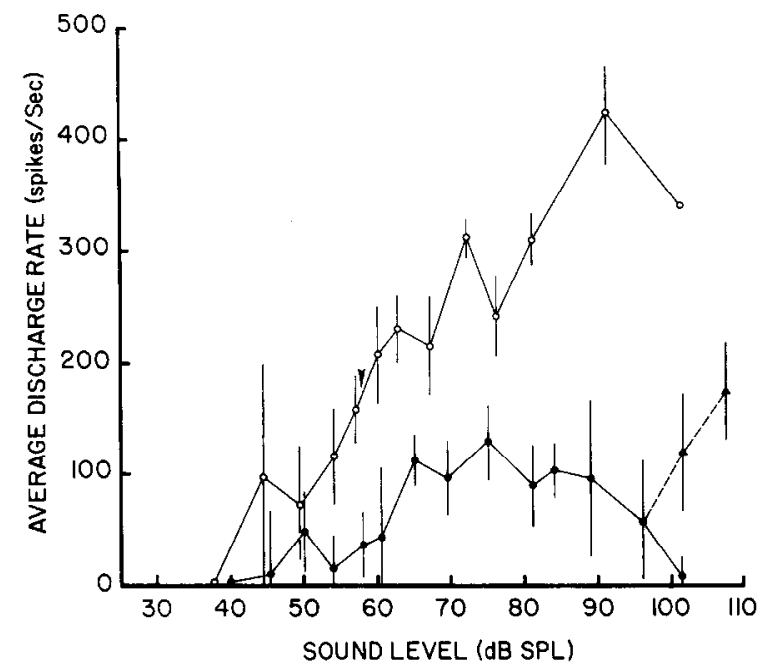

C.

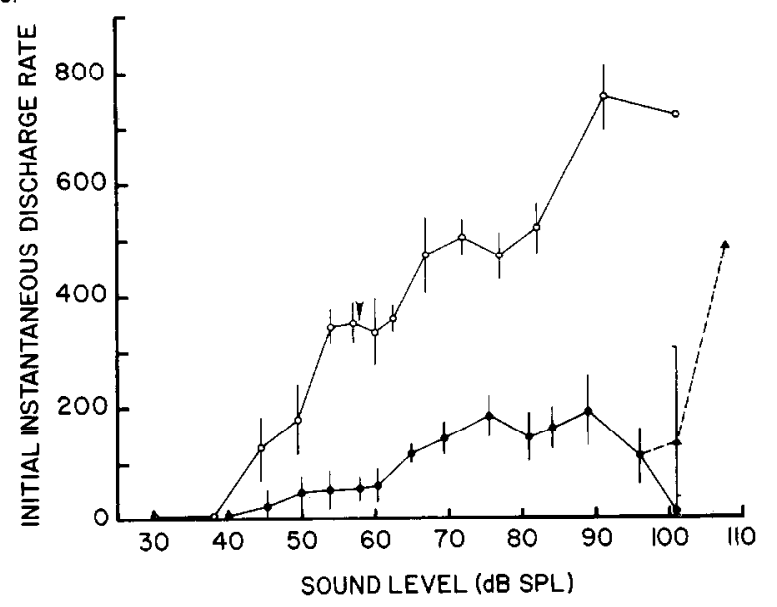

D.

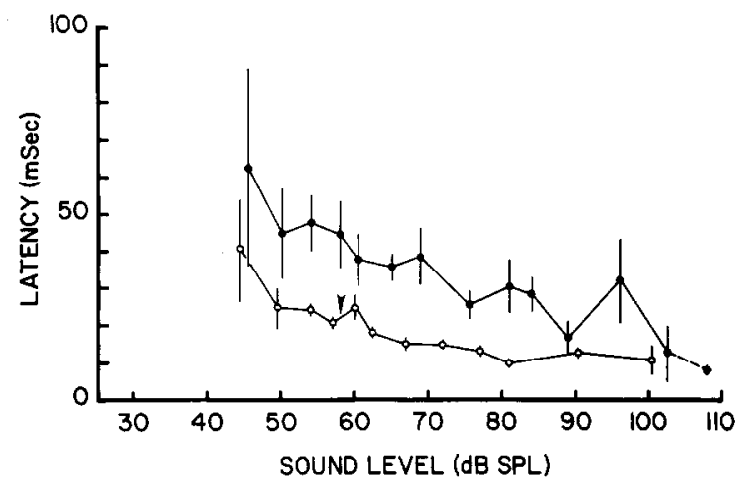

Figure 10. Dynamic range of Int-1 for 5 and $30 \mathrm{kHz}$ tone pulses. The response of Int-1 to 30 -msec-long tone pulses (5 msec rise and fall times) at $5 \mathrm{kHz}$ (solid symbols) and at $30 \mathrm{kHz}$ (open symbols). A, Number of action potentials per tone pulse (measured up to $100 \mathrm{msec}$ after the start of the pulse) versus the sound pressure level of the tone pulse in $\mathrm{dB}$ (SPL). $B$, Average discharge rate versus sound level. $C$, Initial instantaneous discharge rate versus sound level. $D$, Latency of action potential generation versus sound level. Means $\pm 95 \%$ confidence intervals from 11 preparations. Approximately 250 samples were collected for each curve. All preparations were tested up to $91 \mathrm{~dB}$ for $30 \mathrm{kHz}$, but because of technical limitations, only 2 were tested up to $101 \mathrm{~dB}$. At the highest sound levels of $5 \mathrm{kHz}$, most preparations showed breakthrough excitation see text (triangles). The arrowhead indicates the threshold sound level of a 30 -msec-long $30 \mathrm{kHz}$ tone pulse eliciting the negative phonotactic steering response in the behavioral screening tests for the animals represented in this figure.

tory PSPs in Int-1 over a wide range of frequencies. Typically, Int-1 and the other prothoracic interneurons receive a shortlatency EPSP via the ipsilateral ear, followed by contralaterally mediated inhibition (Wohlers and Huber, 1982). However, an important distinguishing characteristic of Int-1 is its short-latency IPSP, which is mediated by sounds near the calling song frequency, rather than by higher frequencies. Ipsilateral inhibition is rapid enough to shunt the rising EPSP, thereby limiting the first $20 \mathrm{msec}$ of response to low discharge rates.

\section{Discharge rate and avoidance steering}

The strong initial inhibition of Int-1, especially during the first $20 \mathrm{msec}$ of response, has implications for initiation of avoidance steering. Several lines of evidence suggest that the initial 20 msec or so of Int-1's response is critical for production of the avoidance behavior. First, Int-1's response to a 30 -msec-long pulse of ultrasound is a rapid burst of action potentials lasting $30-40 \mathrm{msec}$. Nominally, the behavioral response is initiated within $15 \mathrm{msec}$ of the start of Int-1's initial burst: The latency for the steering behavior at threshold ( $50 \mathrm{~dB} \mathrm{SPL})$ is about 40 $\mathrm{msec}$, about $15 \mathrm{msec}$ longer than the latency $(25 \mathrm{msec})$ for action-potential generation in Int- 1 at this same intensity. Interestingly, this relationship holds over $\dot{a}$ wide range of sound levels: the behavioral latency decreases about $15 \mathrm{msec}$, from 40 to $25 \mathrm{msec}$, between 50 and $80 \mathrm{~dB}$ SPL (Fig. 10D); likewise, the latency for action potential generation in Int-1 decreases 15 msec, from 25 to $<10 \mathrm{msec}$, over this same range of intensities (Nolen and Hoy, 1986a; see also Fig. 10). Second, during flight, if intracellular current injection is used to drive Int- 1 to discharge action potentials at a rate above $180 \mathrm{sps}$, avoidance steering is elicited in as little as $30 \mathrm{msec}$ of the first action potential (Nolen and Hoy, 1984; T. G. Nolen, unpublished observations).

In addition, as pulse duration is decreased to $1 \mathrm{msec}$, the burst of action potentials in Int-1 drops to less than $15 \mathrm{msec}$ in duration (Fig. 5); increasing the amplitude of this short pulse by 
A.

$50 \mathrm{~dB}$

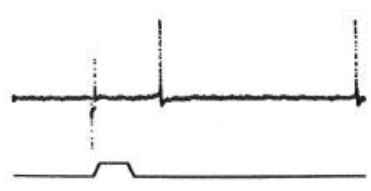

$70 \mathrm{~dB}$

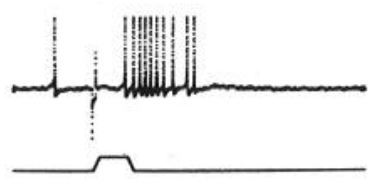

$90 \mathrm{~dB}$

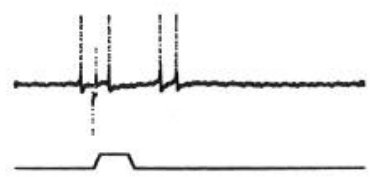

B. $50 \mathrm{~dB}$

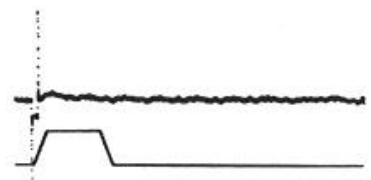

$70 \mathrm{~dB}$

Figure 11. Diversity of Int-1's response to $5 \mathrm{kHz}$ tone pulses $(30 \mathrm{msec}$ long, $5 \mathrm{msec}$ rise and fall times) over the range of sound levels used in Figure 10. $A$, Half of the preparations showed a maximum response at intermediate sound levels, with the response dropping to nearly zero at $100 \mathrm{~dB}$. But note the breakthrough of the lone, short-latency action potential at $80 \mathrm{~dB}$. $B$, Other preparations showed a similar maximum response at intermediate sound levels (about $80 \mathrm{~dB}$ ), with a subsequent decrease at higher sound levels; however, at the highest intensities a large burst of action potentials often breaks through $(100 \mathrm{~dB})$.
$60 \mathrm{~dB}$

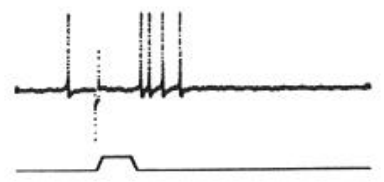

$80 \mathrm{~dB}$

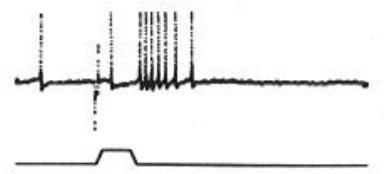

$100 \mathrm{~dB}$

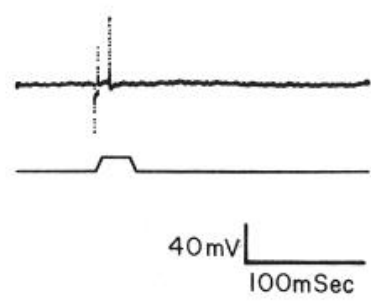

$60 \mathrm{~dB}$

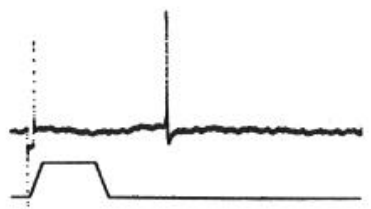

$80 \mathrm{~dB}$

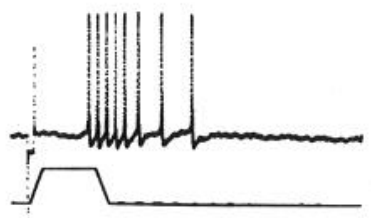

$100 \mathrm{~dB}$

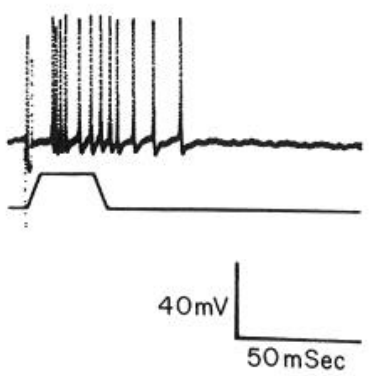

about $10 \mathrm{~dB}$ restores the burst duration to greater than $20 \mathrm{msec}$. This corresponds to the situation in behavioral experiments in which shortening a suprathreshold pulse $(70 \mathrm{~dB}, 30 \mathrm{kHz})$ to less than $1 \mathrm{msec}$ fails to elicit the avoidance behavior (Nolen and Hoy, 1986a); interestingly, the behavior can be reinstated if the pulse amplitude is increased by $10 \mathrm{~dB}$. Thus, while $5 \mathrm{kHz}$ elicits mixed inhibition and excitation, the inhibition is such that it reduces Int-1's initial discharge rate sufficiently to prevent the production of the avoidance behavior, even though the cell may be excited to discharge action potentials. 
A.

\section{$30 \mathrm{kHz} 60 \mathrm{~dB}$}

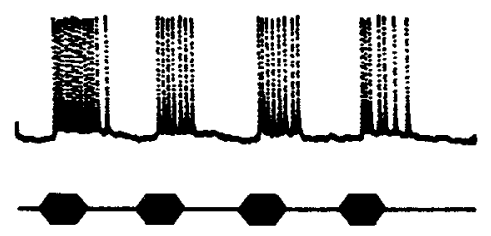

B.

\section{$5 \mathrm{kHz} 70 \mathrm{~dB}$}

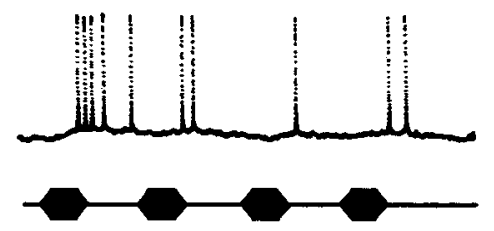

C.

\section{$30 \mathrm{kHz} \quad 70 \mathrm{~dB} \quad 0.5 \mathrm{mSec}$}

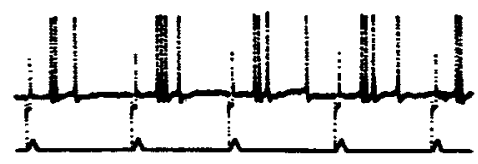

Figure 12. Response of Int-1 to trains of tone pulses. $A$, Response to 30 -msec-long ( $5 \mathrm{msec}$ rise and fall times), $30 \mathrm{kHz}$ tone pulses at 15 pulses/sec. $B$, Response to 30 -msec-long ( $5 \mathrm{msec}$ rise and fall times), 5 $\mathrm{kHz}$ tone pulses at 15 pulses/ $/ \mathrm{sec}$ (an attractive temporal pattern that mediates positive phonotaxis in this species (Pollack, 1982). C, Response to 0.5 -msec-long ( $250 \mu \mathrm{sec}$ rise and fall times), $30 \mathrm{kHz}$ tone pulses at $12 \mathrm{pulses} / \mathrm{sec}$. Calibration marks: $14 \mathrm{mV}$ and $87 \mathrm{msec}$ for $A$ and $B ; 33 \mathrm{mV}$ and $100 \mathrm{msec}$ for $C$.

\section{Acoustic inputs onto Int-1}

The acoustic inputs to Int- 1 are presented schematically in Figure 13. The broad range of frequencies that elicit EPSPs in Int-1 corresponds to the range of sensitivity of broadly tuned afferents that are excited by ultrasound, as well as by the calling song (Hutchings and Lewis, 1981; T. G. Nolen, unpublished observations). These afferents reflect the broad mechanical tuning of the large tympanal membrane (Paton, 1975; Paton et al., 1977; Larsen and Michelsen, 1978; Larsen, 1981) and probably represent the major pathways for low frequency, as well as ultrasonically mediated excitation (Fig. 13).

Low-frequency inhibition reflects the tuning of the majority of afferents in the cricket ear, which are tuned below $10 \mathrm{kHz}$, to the 4-5 kHz carrier frequency of the calling song (Esch et al., 1980; Oldfield et al., 1986). No exclusively ultrasound-sensitive afferents have yet been described. (Perhaps the complex biophysics of the tympanal organ limits the tuning to low-frequency-selective afferents and broadly tuned afferents.) Nevertheless, as shown in Figure 13, low-frequency inhibition of Int-1, combined with broad-band excitation, produces a neuron that has

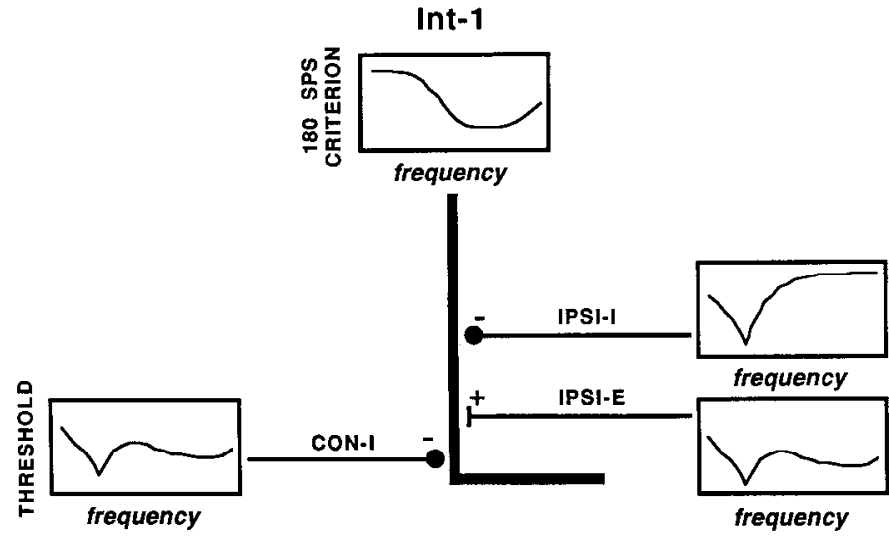

Figure 13. Schematic diagram of the acoustic inputs onto Int-1 resulting in high-frequency selectivity. IPSI-E, ipsilateral broadband excitation; IPSI-I, ipsilateral low-frequency inhibition; $C O N-I$, contralateral broadband inhibition. Ipsilateral excitation is probably via monosynaptic connections from auditory afferents; the source of ipsilateral inhibition is not known. Ipsilaterally mediated broadband excitation and low-frequency inhibition provide high-frequency selectivity. Broadly tuned contralateral inhibition (mediated by ON1) probably provides directional sensitivity (lateral contrast) (e.g., see Selverston et al., 1985)

greater selectivity for ultrasound than do the afferents. As shown (Fig. 13), high frequencies drive the excitatory pathway, producing maximal discharge rates in Int-1. In contrast, low frequencies drive both excitatory and inhibitory pathways, resulting in low discharge rates. Coupled with the requirement for the critical discharge rate of $>180 \mathrm{sps}$, this property ensures that high-frequency (batlike) sounds, rather than calling song, elicit avoidance steering.

Contralateral inhibition is probably mediated by the contralateral ON1 (Wohlers and Huber, 1978, 1982; T. G. Nolen, unpublished observations). [By contralateral, we mean the ON1 receiving its primary excitatory synaptic input from the ear opposite to Int-1's axon; thus, our terminology for ipsilateral/ contralateral is the opposite of that of Selverston et al. (1985).] Selverston et al. (1985) have shown that the selective destruction of an ON1 abolishes contralateral inhibition in AN2 (=Int-1) in $G$. bimaculatus. ON 1 has major output terminals overlapping extensively with Int-1's lateral dendrite (Wohlers and Huber, 1982; T. G. Nolen, unpublished observations), suggesting that contralateral inhibition could result from synaptic inputs from ON1 along Int-1's lateral dendrite. This possibility is supported by intracellular recordings showing that contralateral inhibition, i.e., the late-acting IPSP, is relatively large in the lateral dendrites (Fig. 6C).

The possible neuronal source of ipsilateral (low-frequency) inhibition is not yet known. Measurements of IPSP latencies suggest that both ipsi- and contralateral inhibition are quite rapid (10-15 msec, minimum; compare Figs. $7 B$ and $8 A$ ). However, separate, closed-field stimulation of the 2 ears has shown that ipsilateral inhibition by $5 \mathrm{kHz}$ is functionally more rapid than is contralateral inhibition (T. G. Nolen, unpublished observations), suggesting that the ipsilateral inhibitory synapses may be placed closer to the spike trigger zone, i.e., along the integrating segment, or at the proximal part of the medial or lateral dendrites. However, staining techniques allowing both cell identification and recording site localization must now be used to test these possibilities. 


\section{Dynamic range of response}

As a consequence of inhibition, Int-1 has a small dynamic range for sounds between 3 and $10 \mathrm{kHz}$, where ipsilateral inhibition is strongest. Its response to calling song is relatively poor compared to that of AN1 or ON1 (T. G. Nolen, unpublished observations; see also Wohlers and Huber, 1982).

In contrast, Int- 1 can code the stimulus intensity of ultrasound from $50 \mathrm{~dB}$ up to at least $90 \mathrm{~dB}$ SPL. It can also follow trains of short, batlike pulses of ultrasound (Fig. 12C; see also Moiseff and Hoy, 1983). Flying crickets produce directional steering in response to ultrasound from $55 \mathrm{~dB}$ up to about $90 \mathrm{~dB}$ SPL, where they switch to an evasive behavior similar to that produced by moths at close range to an echolocating bat (Roeder, 1967; Nolen and Hoy, 1986a).

Int-1's wide dynamic range is extraordinary, especially when compared with the ranges of mammalian auditory fibers and cochlear neurons (Møller, 1983). Such a wide dynamic range suggests that Int-1 could function over the range of ultrasonic intensities inherent in predator-prey interactions between flying insects and bats (Miller, 1982, 1983) and could code bat-tocricket distances over the range of $20 \mathrm{~m}$ to $<20 \mathrm{~cm}$, similar to the theoretical distance-detection ability of crickets as determined behaviorally (Nolen and Hoy, 1986a).

The response to calling song suggests that Int-1 would be sensitive to the song over the range of 6 to $10 \mathrm{~cm}$ but that it would not reliably code the song temporal pattern; this would be true especially at higher intensities (shorter distances), where inhibition increases. In addition, the best distance $(10 \mathrm{~cm}-1.6$ $\mathrm{m}$ ) is so short that Int-1 probably would be of limited use during flight when the animal would be far from singing males located on the ground below; however, it might operate over some of the intermediate distances involved in walking phonotaxis. Thus, while it is possible Int- 1 could be important in song recognition and localization, its response properties seem to be optimized for detection and distance coding of batlike ultrasound.

\section{Frequency selectivity}

The physiological responses of identified cricket auditory neurons are consistent with the hypothesis that frequency discrimination is achieved via 2 sets of auditory interneurons. One set, tuned to the calling song fundamental frequency, mediates positive phonotaxis to the calling song (Huber, 1983), while the second set of high-frequency selective neurons, especially Int-1, mediates the avoidance behaviors (Nolen and Hoy, 1984).

Like Int-1, other cricket prothoracic auditory interneurons produce very high discharge rates at their best frequency. For example, the ascending pulse coder AN1 is tuned sharply to the calling song frequency, at which it has its highest discharge rates. ANl discharges at an average rate of $300 \mathrm{sps}$ in response to 70 $\mathrm{dB} 5 \mathrm{kHz}$ tone pulses but only at 170 sps in response to $70 \mathrm{~dB}$ $30 \mathrm{kHz}$ tone pulses. Thus, this cell faithfully codes the calling song temporal pattern but has poorer sensitivity to ultrasound. In comparison, Int- 1 discharges at only 120 sps at $70 \mathrm{~dB} 5 \mathrm{kHz}$ and at $250 \mathrm{sps}$ at $70 \mathrm{~dB} 30 \mathrm{kHz}$. Int-1's poor response to the calling song mirrors AN1's poor response to ultrasound. Thus, frequency selectivity in these 2 channels is mediated by differences in discharge rates. It is tempting to speculate that the high discharge rates in these neurons reflect the requirements for transmission at a "high pass" (or facilitating) synapse and that the postsynaptic cell would not be sufficiently excited if the discharge rate were too low. Similar requirements for naturally high discharge rates in the production of neural correlates of escape behavior have been demonstrated in the wind-sensitive giant interneurons of the cockroach (Ritzmann and Camhi, 1978).

In addition, we emphasize that Int- 1 is selective for high frequencies in terms of a behaviorally relevant, physiological criterion: discharge rate, as determined in a behaving preparation (Nolen and Hoy, 1984). Therefore, the shape of the threshold tuning curve for the avoidance stccring bchavior is probably largely determined by Int- 1 reaching a discharge rate of about $180 \mathrm{sps}$, which is sufficient to produce avoidance steering (Nolen and Hoy, 1984). As shown in Figure $4 C$, Int- 1 discharged above this critical rate only in response to frequencies above $10 \mathrm{kHz}$, a range corresponding to that of the avoidance behavior (Fig. $4 C$ ) and overlapping the range of frequencies contained in batecholocation sonar (Novick, 1977). In contrast, only rarely did sounds near the calling song fundamental cause the cell to discharge at such high rates (Fig. 10B), and then only at very high sound levels when breakthrough excitation occurred. This finding corresponds to behavioral observations that only about $20 \%$ of the animals produced avoidance steering in response to 5 $\mathrm{kHz}$ tone pulses and then only at very high intensities (Nolen and Hoy, 1986a).

\section{Other possible behavioral functions}

An interesting problem arises when a natural calling song is played to the animal. The male cricket's song is not simply a pure tone but contains some higher frequency harmonics, up to $20 \mathrm{kHz}$ (Nolen and Hoy, 1986a) that can excite Int-1 above 180 sps and elicit avoidance steering. Flight phonotaxis experiments have shown that crickets steer away from model calling songs containing only these high frequency "harmonics." Addition of the $5 \mathrm{kHz}$ "fundamental" frequency to the high frequency "harmonic" suppressed the avoidance steering, presumably because the low-frequency inhibition of Int-1 prevented the cell from reaching the critical discharge rate (Nolen and Hoy, 1986b). Two-tone stimulus experiments have shown that tone pulses around $5 \mathrm{kHz}$ suppress the response of Int-1 to high frequencies (Moiseff, 1980; Moiseff and Hoy, 1983; Nolen and Hoy, 1986b). Thus, low-frequency inhibition of Int- 1 could prevent the production of an inappropriate behavior, i.e., negative phonotaxis in response to harmonics of the natural calling song (e.g., see Nolen and Hoy, 1986b).

Our results do not exclude the possibility that Int-1 has several behavioral functions. Clearly, during flight Int-1 can act as a bat-detector, and when stimulated by high frequencies to discharge at rates in excess of 180 sps, it initiates avoidance steering behavior. We hypothesize that initiation of this bchavior requires transmission at a "high pass" synapse in the brain or suboesophageal ganglion (Moiseff and Hoy, 1983) onto descending neurons that drive flight steering (Hoy and Nolen, 1987). Such a filter mechanism permits the animal's nervous system to discriminate between cricket song and batlike sounds by virtue of a discharge rate code in Int-1. In addition, following detection of batlike ultrasound by Int-1, the decision to steer away depends on whether the sound was detected in the context of flight behavior. Previously we found that if the animal was not flying, stimulation of Int-1, even above 180 sps did not elicit motor activity characteristic of avoidance steering (Nolen and Hoy, 1984); the flight oscillator thus appears to gate the command to steer away (Nolen, 1984; Nolen and Hoy, 1984; Hoy and Nolen, 1987). We would like to point out that while the appropriate context for bat-avoidance behavior is flight, it is 
possible that Int-1 also functions in other behaviors and in contexts other than flight. For example, it could also function in species isolation if it is responsible for eliciting active avoidance of the high-frequency songs of other species. Further integrated behavioral and physiological studies should resolve these questions.

\section{References}

Atkins, G., and G. S. Pollack (1986) Anatomy and physiology of sound sensitive descending and $\mathrm{T}$-shaped interneurons in the prothoracic ganglion of the cricket Teleogryllus oceanicus. Soc. Neurosci. Abstr. 12: 858 .

Autrum, H. (1963) Anatomy and physiology of sound receptors in invertebrates. In Acoustic Behavior of Animals, R.-G. Busnel, ed., pp. 412-433, Elsevier, New York.

Boyan, G. S. (1980) Auditory neurones in the brain of the cricket, Gryllus bimaculatus (De Geer). J. Comp. Physiol. 140: 81-93.

Boyan, G. S. (1981) Two-tone suppression of an identified auditory neurone in the brain of the cricket Gryllus bimaculatus (De Geer). J. Comp. Physiol. 144: 117-125.

Boyd, P., and B. Lewis (1983) Peripheral auditory directionality in the cricket (Gryllus campestris L., Teleogryllus oceanicus Le Guillou). J. Comp. Physiol. 153: 523-532.

Boyd, P., R. Kühne, S. Silver, and B. Lewis (1984) Two-tone suppression and song coding by ascending neurones in the cricket Gryllus campestris L. J. Comp. Physiol. A 154: 423-430.

Casaday, G. B., and R. R. Hoy (1977) Auditory interneurons in the cricket Teleogryllus oceanicus: Physiological and anatomical properties. J. Comp. Physiol. 121: 1-13.

Esch, H., F. Huber, and D. W. Wohlers (1980) Primary auditory neurons in crickets: Physiology and central projections. J. Comp. Physiol. 137: 27-38.

Evans, E. F. (1975) Neural processes for the detection of acoustic patterns and for sound localization. In Feature Extraction by Neurons and Behavior, G. Werner, ed., pp. 131-145, MIT Press, Cambridge, MA.

Griffin, D. R., J. H. Friend, and F. A. Webster (1965) Target discrimination by the echolocation of bats. J. Exp. Zool. 158: 155-168.

Hill, K. G. (1974) Carrier frequency as a factor in the phonotactic behavior of female crickets ('ieleogryllus oceanicus). J. Comp. Physiol. 93: 7-18.

Hill, K. G., and G. S. Boyan (1977) Sensitivity to frequency and direction of sound in the auditory system of crickets (Gryllidae). J. Comp. Physiol. 121: 79-97.

Hoy, R. R., and T. G. Nolen (1987) The role of context in decisionmaking by an identified interneuron in the cricket. In Neural and Behavioral Approaches to Higher Brain Functions, S. P. Wise and E. V. Evarts, eds., Wiley-Interscience, New York (in press).

Hoy, R. R., S. Rollins, and G. Casaday (1978) Absence of auditory afferents alters the growth pattern of an identified auditory interneuron. Soc. Neurosci. Absir. 4: 163.

Hoy, R. R., G. S. Pollack, and A. Moiseff (1982) Species-recognition in the field cricket, Teleogryllus oceanicus: Behavioral and neural mechanisms. Am. Lool. 22: 597-607.

Hoy, R. R., T. G. Nolen, and G. B. Casaday (1985) Dendritic sprouting and compensatory synaptogenesis in an identified interneuron following auditory deprivation in a cricket. Proc. Natl. Acad. Sci. USA 82: 7772-7776.

Huber, F. (1983) Neural correlates of Orthopteran and Cicada phonotaxis. In Neuroethology and Behavioral Physiology, F. Huber and $\mathrm{H}$. Markl, eds., pp. 108-135, Springer-Verlag, Berlin.

Hutchings, M., and B. Lewis (1981) Response properties of primary auditory fibers in the cricket Teleogryllus oceanicus (Le Guillou). J. Comp. Physiol. 143: 129-134.

Hutchings, M., and B. Lewis (1984) The role of two-tone suppression of song coding by ventral cord neurones in the cricket Teleogryllus oceanicus (Le Guillou). J. Comp. Physiol. 154: 103-112.

Larsen, O. N. (1981) Mechanical time resolution in some insect ears. II. Impulse sound transmission in acoustic tracheal tubes. J. Comp. Physiol. 143: 297-304.

Larsen, O. N., and A. Michelsen (1978) Biophysics of the Ensiferan ear. III. The cricket as a four-input system. J. Comp. Physiol. 123: $217-227$.
Michelsen, A. (1971a) The physiology of the locust ear. I. Frequency sensitivity of single cells in the isolated ear. Z. Vgl. Physiol. 71: 4962.

Michelsen, A. (1971b) The physiology of the locust ear. III. Acoustical properties of the intact ear. Z. Verlag Physiol. 71: 102-128.

Miller, L. A. (1971) Physiological responses of lacewings (Chrysopa, Neuroptera) to ultrasound. J. Insect Physiol. 17: 491-506.

Miller, L. A. (1982) The orientation and evasive behavior of insects to bat cries. In Exogenous and Endogenous Influences on Metabolic and Neural Control, Vol. 1, A. D. F. Addink and N. Spronk, eds., pp. 393-405, Pergamon; Oxford, U.K.

Miller, L. A. (1983) How insects detect and avoid bats. In Neuroethology and Behavioral Physiology, F. Huber and K. Markl, eds., pp. 25 I266, Springer-Verlag, Berlin.

Moiseff, A. (1980) Auditory interneurons and phonotactic behavior in the Australian field cricket, Teleogryllus oceanicus. Ph.D. thesis, Cornell University, Ithaca, NY.

Moiseff, A., and R. R. Hoy (1983) Sensitivity to ultrasound in an identified auditory interneuron in the cricket: A possible neural link to phonotactic behavior. J. Comp. Physiol. 152: 155-167.

Moiseff, A., G. S. Pollack, and R. R. Hoy (1978) Steering responses of flying crickets to sound and ultrasound: Mate attraction and predator avoidance. Proc. Natl. Acad. Sci. USA 75: 4052-4056.

Møller, A. R. (1983) Auditory Physiology, Academic, New York.

Nolen, T. G. (1984) The neural basis of ultrasound avoidance steering of flying crickets. Ph.D. thesis, Conell University, Ithaca, NY.

Nolen, T. G., and R. R. Hoy (1982) Neural basis of ultrasound avoidance steering in the cricket Teleogryllus oceanicus. Soc. Neurosci. Abstr. 8: 529 .

Nolen, T. G., and R. R. Hoy (1983) Postsynaptic inhibition mediates suppression of ultrasound avoidance in the cricket Teleogryllus oceanicus. Soc. Neurosci. Abstr. 9: 215.

Nolen, T. G., and R. R. Hoy (1984) Initiation of behavior by single neurons: The role of hehavioral context. Science. 226: 992-994.

Nolen, T. G., and R. R. Hoy (1986a) Phonotaxis in flying crickets: I. Attraction to the calling song and avoidance of bat-like ultrasound are discrete behaviors. J. Comp. Physiol. A 159: 423-440.

Nolen, T. G., and R. R. Hoy (1986b) Phonotaxis in flying crickets II. Physiological mechanisms of two-tone suppression of the high frequency avoidance steering behavior by the calling song. J. Comp. Physiol. A 159: 441-456.

Noether, G. (1971) Introduction to Statistics, Houghton Mifflin, New York.

Novick, A. (1977) Acoustic orientation. In Biology of Bats, Vol. 3, W. A. Wimsatt, ed., pp. 74-289, Academic, New York.

Oldfield, B. P. (1982) Tonotopic organisation of auditory receptors in Tettigoniidae (Orthoptera: Ensifera) J. Comp. Physiol. 147: 461-469.

Oldfield, B. P., H.-U. Kleindienst, and F. Huber (1986) Physiology and tonotopic organization of auditory receptors in the cricket Gryllus bimaculatus De Geer. J. Comp. Physiol. A 159: 457-464.

Pallas, S. L., and R. R. Hoy (1986) Regeneration of normal afferent input does not eliminate aberrant synaptic connections of an identified auditory interneuron in the cricket Teleogryllus oceanicus. J. Comp. Neurol. 248: 348-359.

Paton, J. A. (1975) Frequency analysis in the auditory system of field crickets. Ph.D. thesis, Cornell University, Ithaca, NY.

Paton, J. A., K. R. Capranica, P. R. Dragsten, and W. W. Webb (1977) Physical basis for auditory frequency analysis in field crickets (Gryllidae). J. Comp. Physiol. 119: 221-240.

Pollack, G. S. (1982) Sexual differences in cricket calling song recognition. J. Comp. Physiol. 146: 217-221.

Pollack, G. S. (1986) Discrimination of calling song models by the cricket, Teleogryllus oceanicus: The influence of sound direction on neural encoding of the stimulus temporal pattern and on phonotactic behavior. J. Comp. Physiol. A 158: 549-561.

Pollack, G. S., F. Huber, and T. Weber (1984) Frequency and temporal pattern dependent phonotaxis of Teleogryllus oceanicus in flight and walking paradigms. J. Comp. Physiol. 154: 12-26.

Popov, A. V., and A. M. Markovich (1982) Auditory interneurons in the prothoracic ganglion of the cricket Gryllus bimaculatus. II. A highfrequency ascending neurone $\left(\mathrm{HF}_{1} \mathrm{AN}\right)$. J. Comp. Physiol. 146:351359.

Regen, J. (1913) Uber die Anlockung des Weibchens von Gryllus campestris $\mathbf{L}$. Durch telephonisch ubertragene stridulationslaute des Mannchens. Pfluegers Arch. 155: 193-200.

Rheinlaender, J. (1975) Transmission of acoustic information at three 
neural levels in the auditory system of Decticus verrucivorus (Tettigoniidae, Orthoptera). J. Comp. Physiol. 97: 1-53.

Ritzmann, R., and J. H. Camhi (1978) Excitation of leg motor neurons by giant interneurons in the cockroach Periplaneta americana. J. Comp. Physiol. 125: 305-316.

Roeder, K. D. (1967) Nerve Cells and Insect Behavior, Harvard University Press, Cambridge, MA.

Schaller, F., and C. Timm (1950) Das Hörvermögen der Nachtchmetterlinge. Z. Vgl. Physiol. 32: 468-481.

Selverston, A. I., H. U. Kleindienst, and F. Huber (1985) Synaptic connectivity between cricket auditory interneurons as studied by selective photoinactivation. J. Neurosci. 5: 1283-1292.

Simmons, J. A., and S. A. Kick (1983) Interception of flying insects by bats. In Neuroethology and Behavioral Physiology, F. Huber, ed., pp. 267-279, Springer-Verlag, Berlin.

Stephen, R. O., and H. C. Bennet-Clark (1982) The anatomical and mechanical basis of stimulation and frequency analysis in the locust ear. J. Exp. Biol. 99: 279-314.

Stewart, W. W. (1978) Functional connections between cells as revealed by dye-coupling with a highly fluorescent naphthalimide tracer. Cell 14: 741-759.

Stout, J. F., C. H. DeHaan, and R. W. McGhee (1983) Attractiveness of the male Acheta domesticus calling song to females. I. Dependence on each of the calling song features. J. Comp. Physiol. 153: 509-521.
Strausfeld, N. J., N. S. Seyan, D. W. Wohlers, and J. P. Bacon (1983) Lucifer yellow histology. In Functional Neuroanatomy, N.J. Strausfeld, ed., pp. 132-155, Springer-Verlag, Berlin.

Suga, N. (1973) Feature extraction in the auditory system of bats. In Basic Mechanisms of Hearing, A. R. Møller, ed., pp. 675-744, Academic, New York.

Suga, N. (1978) Specialization of the auditory system for reception and processing of species-specific sounds. Fed. Proc. 37: 2342-2354.

Thorson, J., T. Weber, and F. Hubcr (1982) Auditory behavior of the cricket. II. Simplicity of calling song recognition in Gryllus, and anomalous phonotaxis at abnormal carrier frequencies. J. Comp. Physiol. 146: 361-378.

Walker, T. J. (1957) Specificity in the response of female tree crickets (Orthoptera, Gryllidae, Oceanthinae) to calling songs of the males. Ann. Entomol. Soc. Am. 50: 626-636.

Wendler, G., M. Dambach, B. Schmitz, and H. Scharstein (1980) Analysis of the acoustic orientation behaviour in crickets (Gryllus campestris L.). Naturwissenschaften 67: 99-101.

Wohlers, D. W., and F. Huber (1978) Intracellular recording and staining of cricket auditory interneurons. J. Comp. Physiol. 127: 1128.

Wohlers, D. W., and F. Huber (1982) Processing of sound signals by six types of ncurons in the prothoracic ganglion of the cricket Gryllus bimaculatus L. J. Comp. Physiol. 146: 161-173. 